\title{
Disentangling the Galactic Halo with APOGEE. I. Chemical and Kinematical Investigation of Distinct Metal-poor Populations
}

\author{
Christian R. Hayes ${ }^{1}$, Steven R. Majewski ${ }^{1}$ (D), Matthew Shetrone ${ }^{2}$ (D), Emma Fernández-Alvar ${ }^{3}$, Carlos Allende Prieto ${ }^{4,5}$, \\ William J. Schuster ${ }^{6}$, Leticia Carigi ${ }^{3}$, Katia Cunha ${ }^{7,8}$, Verne V. Smith ${ }^{9}$, Jennifer Sobeck ${ }^{10}$, Andres Almeida ${ }^{11}$, \\ Timothy C. Beers ${ }^{12}$ (D) Ricardo Carrera ${ }^{4}$ (D), J. G. Fernández-Trincado ${ }^{13,14}$, D. A. García-Hernández ${ }^{4,5}$, Doug Geisler ${ }^{13}$ (iD, \\ Richard R. Lane $^{15,16}$, Sara Lucatello ${ }^{17}$ (D), Allison M. Matthews ${ }^{1}$ (D), Dante Minniti ${ }^{18,19,20}{ }^{\text {(D) }}$, Christian Nitschelm $^{21}$, \\ Baitian Tang $^{13}$ (D), Patricia B. Tissera ${ }^{18,19}$ (D), and Olga Zamora ${ }^{4,5}$ \\ ${ }^{1}$ Department of Astronomy, University of Virginia, Charlottesville, VA 22904-4325, USA; crh7gs@ virginia.edu \\ ${ }^{2}$ University of Texas at Austin, McDonald Observatory, Fort Davis, TX 79734, USA \\ ${ }^{3}$ Instituto de Astronomía, Universidad Nacional Autónoma de México, Apartado Postal 70-264, México D.F., 04510 México \\ ${ }^{4}$ Instituto de Astrofísica de Canarias (IAC), Vía Láctea, E-38205 La Laguna, Tenerife, Spain \\ ${ }^{5}$ Departamento de Astrofísica, Universidad de La Laguna (ULL), E-38206 La Laguna, Tenerife, Spain \\ ${ }^{6}$ Instituto de Astronomía, Universidad Nacional Autónoma de México, AP 106, 22800, Ensenada, B.C., México \\ ${ }^{7}$ Observatório Nacional, 77 Rua General José Cristino, Rio de Janeiro, 20921-400, Brazil \\ ${ }^{8}$ Steward Observatory, University of Arizona, 933 North Cherry Avenue, Tucson, AZ 85721, USA \\ ${ }^{9}$ National Optical Astronomy Observatories, Tucson, AZ 85719, USA \\ ${ }^{10}$ Department of Astronomy, Box 351580, University of Washington, Seattle, WA 98195, USA \\ ${ }^{11}$ Instituto de Investigación Multidisciplinario en Ciencia y Tecnología, Universidad de La Serena, Benavente 980, La Serena, Chile \\ ${ }^{12}$ Department of Physics and JINA Center for the Evolution of the Elements, University of Notre Dame, Notre Dame, IN 46556, USA \\ ${ }^{13}$ Departamento de Astronomía, Universidad de Concepción, Casilla 160-C, Concepción, Chile \\ ${ }^{14}$ Institut Utinam, CNRS UMR6213, Univ. Bourgogne Franche-Comté, OSU THETA, Observatoire de Besançon, BP 1615, F-25010 Besançon Cedex, France \\ ${ }^{15}$ Millennium Institute of Astrophysics, Av. Vicuña Mackenna 4860, 782-0436 Macul, Santiago, Chile \\ ${ }^{16}$ Instituto de Astrofísica, Pontificia Universidad Católica de Chile, Av. Vicuña Mackenna 4860, 782-0436 Macul, Santiago, Chile \\ ${ }^{17}$ Osservatorio Astronomico di Padova-INAF, Vicolo dell'Osservatorio 5, I-35122, Padova, Italy \\ ${ }^{18}$ Departamento de Ciencias Físicas, Facultad de Ciencias Exactas, Universidad Andrés Bello, Av. Fernández Concha 700, Las Condes, Santiago, Chile \\ ${ }^{19}$ Instituto Milenio de Astrofísica, Av. Vicuna Mackenna 4860, Macul, Santiago, Chile \\ ${ }^{20}$ Vatican Observatory, V00120 Vatican City State, Italy \\ ${ }^{21}$ Unidad de Astronomía, Universidad de Antofagasta, Avenida Angamos 601, Antofagasta 1270300, Chile \\ Received 2017 August 2; revised 2017 November 15; accepted 2017 November 15; published 2018 January 5
}

\begin{abstract}
We find two chemically distinct populations separated relatively cleanly in the $[\mathrm{Fe} / \mathrm{H}]-[\mathrm{Mg} / \mathrm{Fe}]$ plane, but also distinguished in other chemical planes, among metal-poor stars (primarily with metallicities $[\mathrm{Fe} / \mathrm{H}]<-0.9$ ) observed by the Apache Point Observatory Galactic Evolution Experiment (APOGEE) and analyzed for Data Release 13 (DR13) of the Sloan Digital Sky Survey. These two stellar populations show the most significant differences in their $[\mathrm{X} / \mathrm{Fe}]$ ratios for the $\alpha$-elements, $\mathrm{C}+\mathrm{N}, \mathrm{Al}$, and $\mathrm{Ni}$. In addition to these populations having differing chemistry, the low metallicity high-Mg population (which we denote "the HMg population") exhibits a significant net Galactic rotation, whereas the low-Mg population (or "the LMg population") has halo-like kinematics with little to no net rotation. Based on its properties, the origin of the $\mathrm{LMg}$ population is likely an accreted population of stars. The $\mathrm{HMg}$ population shows chemistry (and to an extent kinematics) similar to the thick disk, and is likely associated with in situ formation. The distinction between the $\mathrm{LMg}$ and HMg populations mimics the differences between the populations of low- and high- $\alpha$ halo stars found in previous studies, suggesting that these are samples of the same two populations.
\end{abstract}

Key words: Galaxy: disk - Galaxy: evolution - Galaxy: formation - Galaxy: halo - stars: abundances

Supporting material: machine-readable table

\section{Introduction}

A key step to reconstructing the history of the Milky Way's (MW) formation and evolution is to identify and characterize its constituent stellar populations. Metal-poor stars probe the early evolution of the Galaxy and give clues to the origin of its first components. Among the MW components containing a large fraction of metal-poor stars are the thick disk (originally known as Intermediate Pop II stars and later reidentified by Yoshii 1982; Gilmore \& Reid 1983) via its metal-poor extension (the metal-weak thick disk, MWTD, Morrison 1993; Chiba \& Beers 2000; Beers et al. 2002), globular clusters, dwarf MW satellite galaxies, and the halo, possibly separating into inner- and outer-halo components (Hartwick 1987; Sommer-Larsen \& Zhen 1990; Allen et al. 1991; Kinman et al. 1994; Norris 1994; Carollo et al. 2007, 2010; Beers et al. 2012), but containing sub-populations of globular clusters (Zinn 1993) and fields stars accreted from hierarchical formation, which undoubtedly played a key role in the formation of the halo. A long standing problem is whether and how these different populations may be discriminated from one another by their spatial, kinematical, and chemical distributions.

A commonly used strategy is to rely on kinematical definitions to separate stars into populations (Venn et al. 2004; Reddy et al. 2006; Ruchti et al. 2011; Ishigaki et al. 2012, 2013). Unfortunately, this is fraught with several difficulties, not least that it requires that the necessary kinematical data are in hand and of sufficient quality to 
provide meaningful discrimination. More problematic than these practical requirements is that the kinematical distributions of these various Galactic components typically overlap so that it is generally not possible to undertake definitive separations of stars into their respective populations with kinematical information alone. Even resorting to simple statistical prescriptions can be perilous given uncertainties in critical priors used to define and fit distribution functions, such as the number of components to fit (see the discussion in Carollo et al. 2010) and their intrinsic shapes (not necessarily Gaussian) and therefore free parameters.

Nonetheless, studies of the detailed chemistry of kinematically defined populations have successfully revealed some of the primary chemical characteristics of these metal-poor populations. The chemical properties of the thick disk and at least some subset of the halo, although not always cleanly distinct but showing overlaps, have been shown to exhibit demonstrably different mean chemistry for numerous chemical elements (Nissen \& Schuster 1997, 2010, 2011; Ishigaki et al. 2012, 2013; Ramírez et al. 2012). For example, these studies have shown that at least some fraction of halo stars have lower abundances of $\alpha$-elements (particularly $\mathrm{O}, \mathrm{Mg}$, and $\mathrm{Si}$ ), $\mathrm{Na}, \mathrm{Ni}$, $\mathrm{Cu}$, and $\mathrm{Zn}$ and higher Eu enrichement than those of the thick disk at metallicities $[\mathrm{Fe} / \mathrm{H}] \gtrsim-1.5$.

One early study of the detailed chemical abundances of 29 metal-poor stars suggested that there may be two chemical abundance patterns among halo stars, one of which differed from the thick disk abundance pattern (Nissen \& Schuster 1997). In a subsequent study of an enlarged sample of 94 stars with metallicities $-1.6<[\mathrm{Fe} / \mathrm{H}]-0.4$, Nissen \& Schuster (2010) used chemical abundances to resolve two rather distinct and mostly non-overlapping populations of stars with halo-like kinematics in the $[\mathrm{Mg} / \mathrm{Fe}]-[\mathrm{Fe} / \mathrm{H}]$ chemical plane, with one population having chemistry consistent with the thick disk and the other distinctly less Mg-enriched. Among differences in $\mathrm{Mg}$ and other $\alpha$-elements (distinguishable as "high- and low- $\alpha$ " halo star groupings), these two metal-poor populations were shown to have different abundances in many of the odd- $Z$ and heavier elements listed above (Nissen \& Schuster 2010, 2011; Navarro et al. 2011; Ramírez et al. 2012; Sheffield et al. 2012; Jackson-Jones et al. 2014; Hawkins et al. 2015).

Furthermore, using isochrone fits to stellar surface temperatures and gravities, these two $[\alpha / \mathrm{Fe}]$ groupings were shown to exhibit different mean ages, with the low- $\alpha$ population being younger (Schuster et al. 2012). From the $\alpha$-element abundances and kinematics of the two populations, these past studies have suggested that the low- $\alpha$ population has been accreted through the mergers of dwarf spheroidal-like galaxies (an origin also suggested for "young halo" globular clusters; see Zinn 1993), whereas the high- $\alpha$ stars were likely formed in situ or have been kicked out from the disk (Sheffield et al. 2012; Johnston 2016). Recent studies have also revealed low- $\alpha$ bulge stars, most of which are thought to be chemically associated with the thin disk (Recio-Blanco et al. 2017). While most of these low- $\alpha$ bulge stars have $\alpha$-element abundances that seem too high to be associated with the low- $\alpha$ halo population, a few of these "bulge stars" may have chemical abundances more similar to the low- $\alpha$ halo population. It would not be surprising if low- $\alpha$ halo stars were found in the bulge, since the densities of other stellar populations increase toward the center of the Galaxy.
Despite the proven utility of high precision, high-resolution spectroscopic measurements of chemical abundances to distinguish chemically distinct populations of metal-poor stars, such work is observationally expensive. Consequently, previous sample sizes have generally been limited to a few hundred metal-poor stars (as in the references above). However, the advent of systematic high-resolution surveys, such as the APOGEE survey (Apache Point Observatory Galactic Evolution Experiment; Majewski et al. 2017), the Gaia-ESO Survey (Gilmore et al. 2012), and the GALAH survey (Galactic Archaeology with HERMES; De Silva et al. 2015), brings the opportunity to put these types of studies on a much firmer statistical footing. In this work, we use data from the APOGEE survey to gain a more comprehensive view of the chemical differences between populations of metal-poor stars.

The APOGEE-1 survey (Majewski et al. 2017) observed $\sim 146,000$ stars with good quality ( $/ \mathrm{N} \geqslant 100$ ), high-resolution $(R \sim 22,500)$, infrared $(1.5-1.7 \mu \mathrm{m})$ spectra from which abundances have been derived for up to 23 elemental species in Data Release 13 (DR13; Albareti et al. 2017), at least for more metal-rich and cool stars (Holtzman et al. 2015). Because metal-poor stars are relatively rare and APOGEE, for the most part, uses no special pre-selection for them, they comprise a relatively small fraction of the APOGEE sample. Nevertheless, the APOGEE-1 sample (according to abundances derived for DR13) includes over 1000 metal-poor stars having $[\mathrm{Fe} / \mathrm{H}]<-1.0$ extending down to $[\mathrm{Fe} / \mathrm{H}] \sim-2.0$ (i.e., a sample several times larger than previous studies) and with reliable chemical abundances for as many as 12 elements. Such a large sample of metal-poor stars and a highly dimensional chemical space enables robust searches for chemically distinct metal-poor populations and allows testing of previous claims with a larger statistical footing. Moreover, because the main APOGEE survey targets are only selected photometrically, APOGEE-based studies are free of kinematical biases and include stars from a much larger volume of the Galaxy than previous studies, especially those restricted to observing nearby stars with measured proper motions (e.g., Reddy et al. 2006; Nissen \& Schuster 2010, 2011; Ishigaki et al. 2012, 2013; Bensby et al. 2014).

This work differs from the past study of metal-poor field stars with APOGEE by Hawkins et al. (2015) in the lack of any kinematical selection and use of data driven chemical identification of distinct chemical populations that is supported by independent statistical clustering analyses. In addition, we use APOGEE data from DR13, which, through improvements to the data reduction, stellar parameter, and chemical abundance pipelines has improved APOGEE's chemical abundances and provided a much larger sample of metal-poor stars with accurate chemical abundances compared to that provided by DR12, used by Hawkins et al. (2015). The DR13 improvements to chemical abundance measurements, in particular, allow us greater power to statistically discriminate and characterize the population of proposed low- $\alpha$ accreted halo stars noticed in previous studies from the population of metal-poor stars having higher $\alpha$-element abundances.

We show that the $[\mathrm{Mg} / \mathrm{Fe}]-[\mathrm{Fe} / \mathrm{H}]$ chemical plane is an especially powerful and reliable diagnostic for this population analysis, and one readily provided by APOGEE for the majority of stars, while other element ratios, like $[\mathrm{Al} / \mathrm{Fe}]$ and $[(\mathrm{C}+\mathrm{N}) / \mathrm{Fe}]$, are equally discriminating, if less available for all 
stars, $([(\mathrm{C}+\mathrm{N}) / \mathrm{Fe}]$ becomes uncertain at the lowest metallicities in our study; see Section 3.2 for a discussion of the limitations of the $\mathrm{C}$ and $\mathrm{N}$ abundances). We provide evidence supporting our new selection criteria in these and other chemical dimensions by presenting the results of multidimensional clustering algorithms on the APOGEE-observed metal-poor stars. Moreover, because our sample is kinematically unbiased, we can more reliably explore and characterize the kinematical properties of these chemical groupings; we show that the two primary $[\mathrm{Mg} / \mathrm{Fe}]$-based metal-poor groupings have decidedly different kinematical properties that give clues to their origin and relation to the main spatio-kinematical populations of the Galaxy.

In particular, as suggested by previous work, the high-Mg population is relatively kinematically cold and rotating while the low-Mg population has hot kinematics consistent with expectations for an accreted population. Finally, because metalpoor stars characterized by low- $\alpha$ abundance patterns are traditionally attributed to satellite accretion, we compare the detailed chemical properties of our $\mathrm{Mg}$ populations to those in MW satellites.

This paper is organized as follows. In Section 2, we discuss the data and selection criteria employed to create the parent stellar sample used throughout the paper. In Section 3, we first discuss our identification of two populations of metalpoor stars based on their $[\mathrm{Mg} / \mathrm{Fe}]$. We then examine the chemical signatures of these populations in other dimensions of APOGEE-observed chemical space, and apply multidimensional clustering algorithms to further justify our characterization of these metal-poor populations. Section 3 also presents the kinematical properties of these populations derived from APOGEE radial velocity data. In Section 4, we compare our sample of stars and the populations we have defined to those suggested and discussed in previous studies. We also comment on the possible origins of these populations, aided by a comparison of our data to the abundance patterns of MW satellites. We present our conclusions in Section 5. A companion paper, Fernández-Alvar et al. (2017b), further explores the chemical evolution and star formation histories of the two populations discriminated in this work.

\section{Data}

Using the Sloan $2.5 \mathrm{~m}$ telescope at Apache Point Observatory (Gunn et al. 2006) as a part of the third installment of the Sloan Digital Sky Survey (SDSS-III, Eisenstein et al. 2011), APOGEE spectroscopically observed a relatively homogenous sample of $\sim 146,000 \mathrm{MW}$ stars to survey its multiple structural components. The details of the APOGEE instrument, survey, data, and calibration are outlined in Majewski et al. (2017) and references therein. Here we present an analysis of the APOGEE data presented in the SDSS Data Release 13 (DR13; Albareti et al. 2017), the first data release of SDSS-IV (Blanton et al. 2017). In this data release, a reanalysis of the spectra presented in SDSS DR12 was performed to improve the quality of derived parameters. Target selection and data reduction for APOGEE are described in detail by Zasowski et al. (2013) and Nidever et al. (2015), respectively, and the APOGEE Stellar Parameter and Chemical Abundances Pipeline (ASPCAP; for a detailed description see García Pérez et al. 2016) was used to determine the stellar parameters and chemical abundances from the best fits to pre-computed libraries of synthetic stellar spectra (e.g., Zamora et al. 2015).
We restrict our analysis to a subsample of stars selected on the basis of a series of APOGEE flags and other constraints. We first removed any stars with the STARFLAGS BAD_PIXELS, VERY_BRIGHT_NEIGHBOR, or LOW_SNR flags set. We also cut any stars with the following ASPCAPFLAGS: METALS_WARN, ROTATION_WARN, METALS_BAD, STAR_BAD, ROTATION_BAD, or SN_BAD. Descriptions of these flags can be found online in the SDSS DR13 bitmask documentation. ${ }^{22}$

In addition to trimming the APOGEE data set using quality flags, we also require that the visit-to-visit velocity scatter be small, i.e., $V_{\text {scatter }} \leqslant 1 \mathrm{~km} \mathrm{~s}^{-1}$, because a larger velocity scatter may indicate surface activity, the presence of a companion, or other astrophysical complications that may make determined parameters and abundances less reliable. Similarly, we only use stars with velocity uncertainties $V_{\text {err }} \leqslant 0.2 \mathrm{~km} \mathrm{~s}^{-1}$, to exclude stars with large velocity uncertainties that may have less reliably derived parameters. We have also restricted our analysis to stars with surface temperatures $4000 \mathrm{~K}<$ $T_{\text {eff }}<5500 \mathrm{~K}$ (selected before applying the post-calibration corrections to produce the surface temperatures and gravities listed in Table 1), given that, as of DR13, ASPCAP is not yet tuned for reliable parameter estimation for cooler stars or those that are warmer and have weaker lines.

Finally, because we will be primarily concerned with magnesium abundances, we only select stars with $\sigma[\mathrm{Mg} / \mathrm{Fe}]<0.1$ and $\mathrm{S} / \mathrm{N}>100$. However, in considering other elemental abundances throughout the remainder of the paper, we only examine (but do not remove from the sample) stars with uncertainties on those abundances below $0.1 \mathrm{dex}$ as well. Because globular clusters are known to exhibit high levels of self-enrichment (Gratton et al. 2004), we have excluded those cluster stars that are easily distinguished from field stars and that can be associated with specific globular clusters (based on proximity, radial velocities, and metallicities). The Sagittarius dwarf spheroidal galaxy ( $\mathrm{Sgr} \mathrm{dSph}$ ) is the only dSph present in DR13, so in addition to removing globular cluster members, we also removed known Sgr dSph members. While the coolest, most luminous $\mathrm{Sgr} \mathrm{dSph}$ giants are removed by our $T_{\text {eff }}>4000 \mathrm{~K}$ requirement, we also removed any stars with the TARGFLAG APOGEE_SGR_DSPH, which was assigned to known Sgr dSph members. After all of these quality cuts and the exclusion of globular cluster and Sgr dSph stars, we are left with 61,742 stars for study using the calibrated ASPCAP stellar parameters and chemical abundances.

\section{Results and Analysis}

\subsection{Two Populations Seen in $[\mathrm{Mg} / \mathrm{Fe}]$ Ratios of Metal-poor Stars}

Visual inspection of the elemental abundances observed by APOGEE for metal-poor stars revealed the most apparent and distinct bimodality in the distribution of $[\mathrm{Mg} / \mathrm{Fe}]$. We therefore first present and examine the distribution of $\mathrm{Mg}$ abundances. This strategy is also motivated by previous studies of metalpoor stars that also found two distinct metal-poor groups based on their $[\alpha / \mathrm{Fe}]$ ratios, or specifically their $[\mathrm{Mg} / \mathrm{Fe}]$ ratios (see Section 1).

Figure 1 shows the distribution of $[\mathrm{Mg} / \mathrm{Fe}]$ with $[\mathrm{Fe} / \mathrm{H}]$ for all stars that made it through the quality criteria discussed in Section 2. Most obvious in this plot are the high- and

\footnotetext{
22 http://www.sdss.org/dr13/algorithms/bitmasks/
} 
Table 1

Properties, Parameters, and Population Identification of APOGEE DR13 Metal-poor Stars

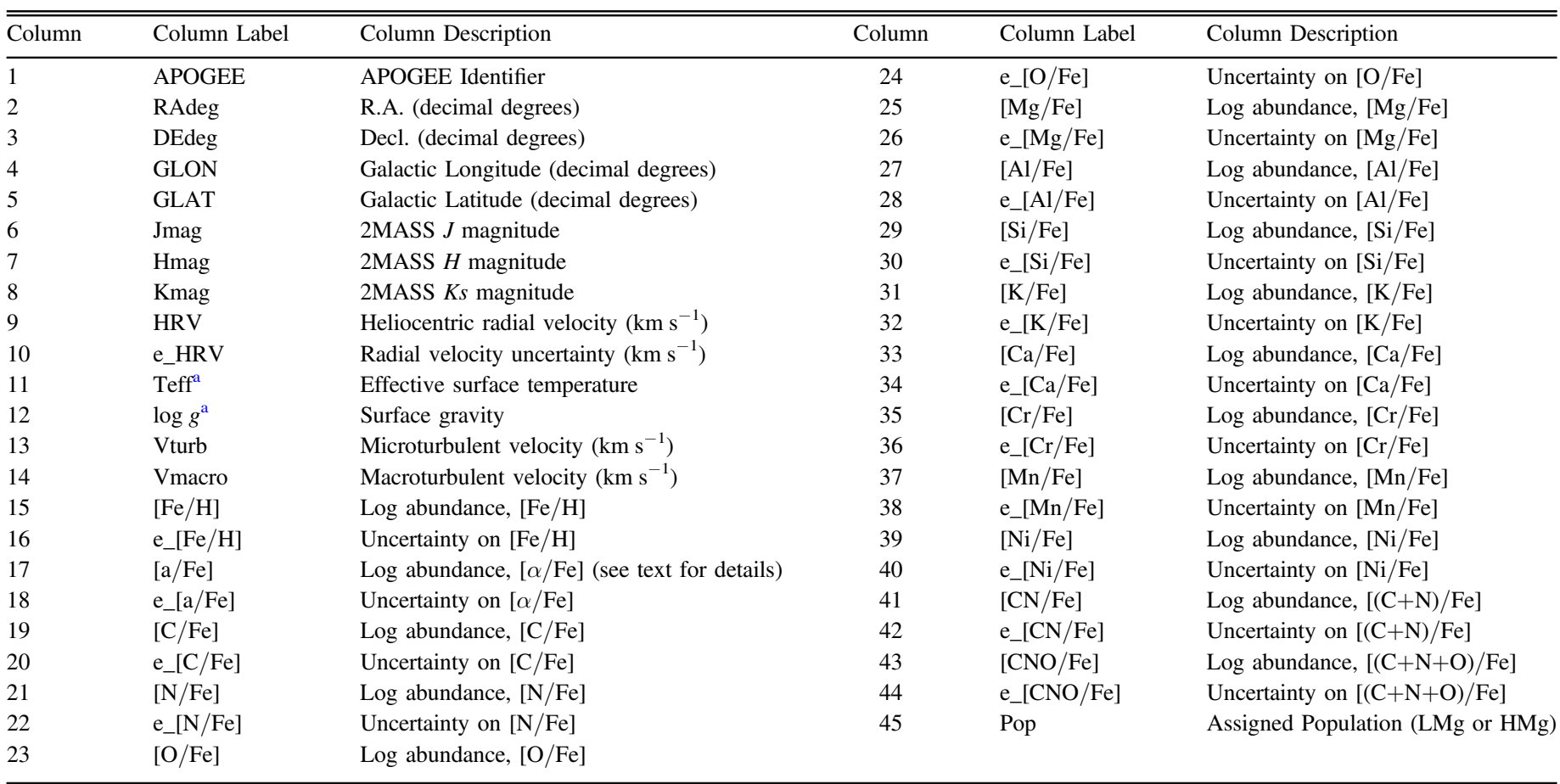

Note. Null table entries are given values of -9999 .

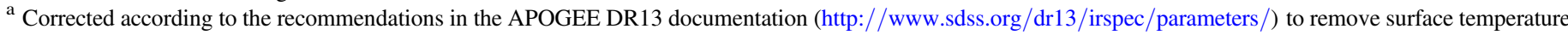
and gravity trends found post-calibration.

(This table is available in its entirety in machine-readable form.)

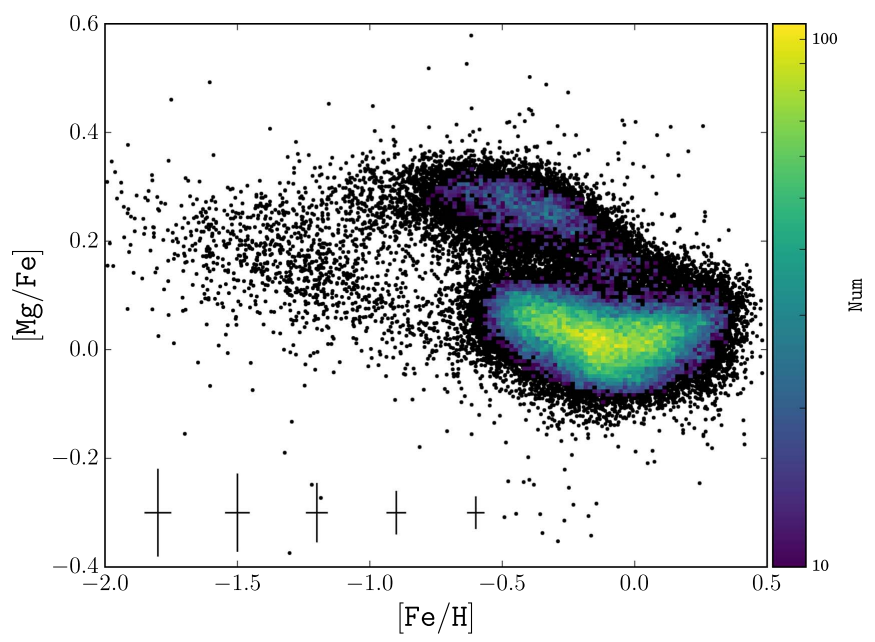

Figure 1. Distribution of $[\mathrm{Mg} / \mathrm{Fe}]$ with metallicity for the APOGEE DR13 stars surviving the quality cuts discussed in Section 2. A $2 \mathrm{D}$ histogram is plotted for the highly populated portions of this chemical space (corresponding to the chemical domain of the relatively more metal-rich thin and thick disk populations), while individual stars are plotted where APOGEE-observed stars are less populous in this plane. The plotted error bars show the median abundance uncertainties in 0.3 dex wide metallicity bins. In addition to the traditional thick disk sequence seen at $[\mathrm{Fe} / \mathrm{H}]>-1.0$, a noticeable third sequence of stars appears between the low metallicity end of our sample and $[\mathrm{Fe} / \mathrm{H}] \sim-1.0$, with decreasing $[\mathrm{Mg} / \mathrm{Fe}]$ with increasing metallicity.

low- $[\mathrm{Mg} / \mathrm{Fe}]$ tracks between $[\mathrm{Fe} / \mathrm{H}] \sim-0.9$ and +0.4 nominally corresponding to the thick and thin disks respectively. In the APOGEE DR13 data, the high- $\alpha$ sequence commonly associated with the thick disk (e.g., Bovy et al. 2016, and

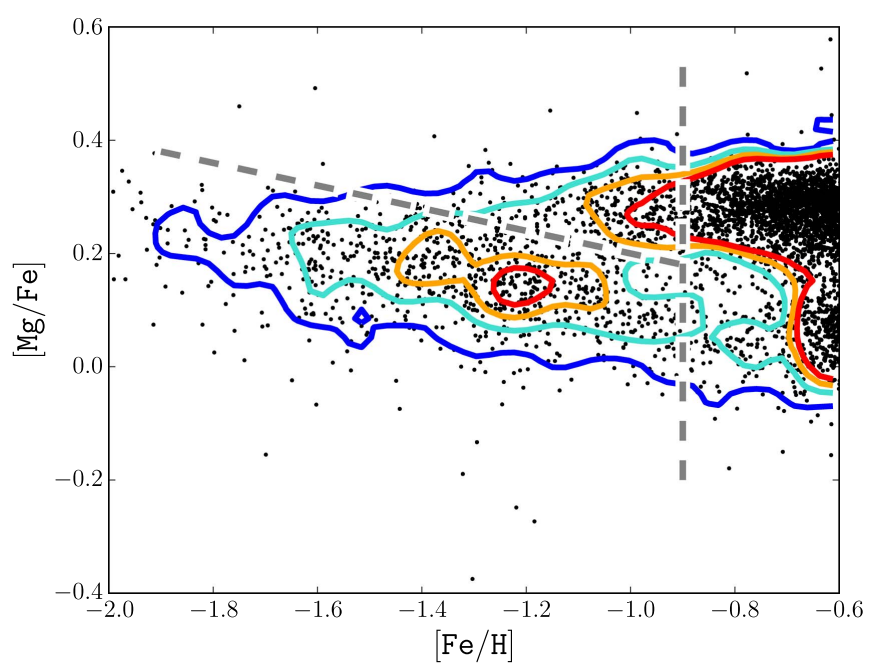

Figure 2. Magnified portion of the metal-poor region of Figure 1, with contours showing the density of stars in the metal-poor regions of Figure 1 . The contours are at $5,15,25$, and 35 stars per $0.0039 \mathrm{dex}^{2}$. These contours demonstrate that there is a low density valley separating two higher density regions, one with lower $[\mathrm{Mg} / \mathrm{Fe}]$, and one with higher $[\mathrm{Mg} / \mathrm{Fe}]$ that appears to be a metal-poor extension of the thick disk locus. The sloping dashed line is adopted to separate the two populations based on their $[\mathrm{Mg} / \mathrm{Fe}]$ and metallicity in Section 3.1.

references therein) seems to taper off at low metallicities, and there appears to be a gap between the thick disk and another set of stars with not only a lower level of $[\mathrm{Mg} / \mathrm{Fe}]$, but decreasing $[\mathrm{Mg} / \mathrm{Fe}]$ with increasing metallicity. 


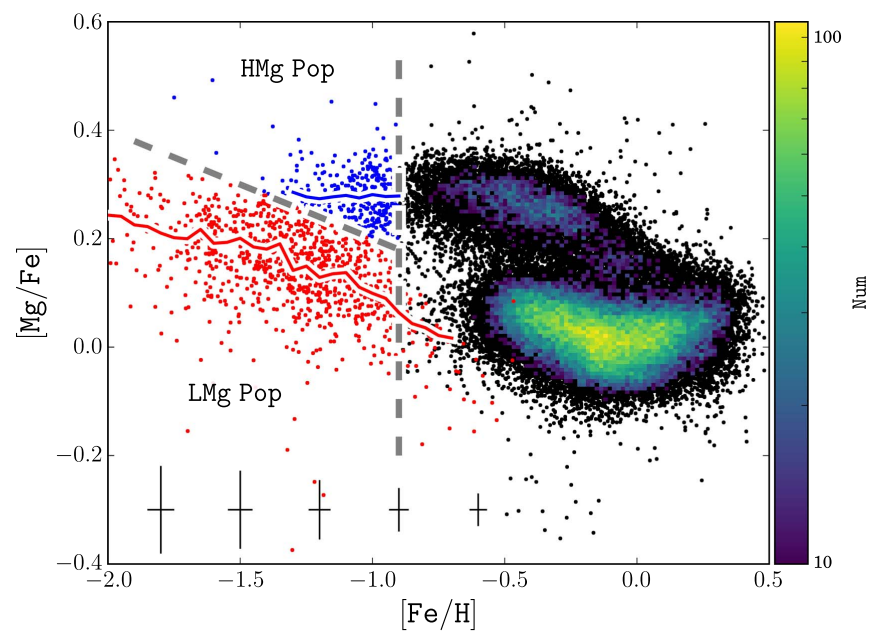

Figure 3. Same as in Figure 1, but with the initial division to separate the relatively $\mathrm{Mg}$-poor population $\mathrm{LMg}$ (red) and the $\mathrm{Mg}$-rich population $\mathrm{HMg}$ (blue). Stars in the $\mathrm{LMg}$ population with metallicities $[\mathrm{Fe} / \mathrm{H}]>-0.9$ have been selected based on their $[(\mathrm{C}+\mathrm{N}) / \mathrm{Fe}]$ abundance ratios, since they appear to follow the abundance pattern of the $\mathrm{LMg}$ population as discussed in Section 3.2

. Overplotted are the roving boxcar medians of the 50 nearest neighbors, again color-coded by population

This gap is made more apparent in Figure 2, where we plot density contours over our data to demonstrate that there is a true low density valley separating the two sequences of metalpoor stars. The peak-to-valley ratio between the density along the $\mathrm{Mg}$-poor sequence and the density in the valley, tracked by the sloped dashed line in Figure 2, gives us an idea of the significance of this second, low-Mg abundance sequence. At a metallicity of $[\mathrm{Fe} / \mathrm{H}] \sim-1.5$ the peak-to-valley ratio is about 1.5 , increasing to 2.5 at a metallicity of $[\mathrm{Fe} / \mathrm{H}] \sim-1.2$, and it is highest at about 3.0 around a metallicity of -1.0 . This indicates that the valley separating these two sequences is more significant at higher metallicities, where the chemical separation between this sequence and the nominal thick disk is larger. It also highlights that at lower metallicities the two low metallicity sequences overlap more, so that it is more difficult to separate them.

Thus, at lower metallicities $([\mathrm{Fe} / \mathrm{H}] \lesssim-0.9)$ the APOGEE data strongly suggest the existence of two populations of stars chemically differentiated by their $[\mathrm{Mg} / \mathrm{Fe}]$ patterns. For simplicity, we initially separate the two populations along the gap or valley by $[\mathrm{Mg} / \mathrm{Fe}]=-0.2 \times[\mathrm{Fe} / \mathrm{H}]$, as shown by the sloped dashed line in Figure 2. We designate the low- $[\mathrm{Mg} / \mathrm{Fe}]$ population as the Low-Mg ( $\mathrm{LMg}$ ) population and the high$[\mathrm{Mg} / \mathrm{Fe}]$ magnesium population as the high- $\mathrm{Mg}(\mathrm{HMg})$ population. For this analysis, we initially restrict our analysis to metallicities of $[\mathrm{Fe} / \mathrm{H}] \leqslant-0.9$, to avoid contaminating the $\mathrm{LMg}$ population with stars from the thin disk locus. Note, however, that we show below in Section 3.2 that the $\mathrm{LMg}$ population extends to slightly higher metallicities as seen by the consideration of $[(\mathrm{C}+\mathrm{N}) / \mathrm{Fe}]$ ratios. Our initial division of the $\mathrm{LMg}$ and $\mathrm{HMg}$ for $[\mathrm{Fe} / \mathrm{H}] \leqslant-0.9$ is shown in Figure 3. In Table 1, we report the relevant properties, stellar parameters, and chemical abundances of the stars categorized into these two populations.

Given that the gap between these populations is not completely devoid of stars, there is some uncertainty in separating them, and there is likely to be some spread of each population across the adopted division, whether due to intrinsic scatter of the true underlying populations or to measurement uncertainties, leading to some low level of cross-contamination that appears to become more significant at lower metallicities $([\mathrm{Fe} / \mathrm{H}] \sim-1.3)$ where the sequences begin to merge. This is examined in more detail using the full chemical profiles of these populations and clustering algorithms in Section 3.3. Because the HMg population smoothly connects with the thick disk locus, this population is likely the metal-weak extension of the thick disk. The origin of the $\mathrm{LMg}$ population is not immediately clear and is examined in more detail in later sections.

\subsection{Chemical Signatures}

While we have identified a potential division in populations with low metallicities in $[\mathrm{Mg} / \mathrm{Fe}]-[\mathrm{Fe} / \mathrm{H}]$ space, for it to have astrophysical significance, we expect it should be revealed in additional dimensions, which we now examine. In the following subsections, we present and analyze the chemical distributions of our sample in other element planes, restricting our analysis of each element to the stars with uncertainties $\sigma[\mathrm{X} / \mathrm{Fe}]<0.1 \mathrm{dex}$ in that element alone.

\subsection{1. $\alpha$-elements: $O, S i$, and $\mathrm{Ca}$}

We first examine other $\alpha$-elements measured by APOGEE with high precision: $\mathrm{O}, \mathrm{Si}$, and $\mathrm{Ca}$, as well as the ASPCAP global $\alpha$-element parameter (derived from the initial ASPCAP fit to all $\alpha$-elements, $\mathrm{O}, \mathrm{Mg}, \mathrm{Si}, \mathrm{Ca}, \mathrm{S}$, and $\mathrm{Ti}$; see Holtzman et al. 2015), whose abundances relative to $\mathrm{Fe}$ are shown in Figure 4 . $\mathrm{Ti}$ is a commonly studied $\alpha$-element and is measured by APOGEE; however, it is considered unreliable because it is not able to reproduce the $[\mathrm{Ti} / \mathrm{Fe}]$ bimodality seen in solar neighborhood studies or in other $\alpha$-element abundances measured by APOGEE (Holtzman et al. 2015). The inconsistency with optically derived $\mathrm{Ti}$ abundances may be due to the ASPCAP inclusion of lines affected by NLTE or saturation (Hawkins et al. 2016) in the measurement of Ti, or a high $T_{\text {eff }}$ sensitivity of $H$-band TiI lines (Souto et al. 2016), regardless of the cause, because of this unreliability, we do not analyze Ti here.

Reassuringly, we find that the $\mathrm{LMg}$ population is consistently lower in $\mathrm{O}, \mathrm{Si}$, and $\mathrm{Ca}$ abundances and the $\mathrm{HMg}$ population is higher, as seen in magnesium; however, the separation between the $\mathrm{LMg}$ and $\mathrm{HMg}$ populations is not as clean in these other $\alpha$-elements as it is in magnesium. The potential exception to this is perhaps in the total $\alpha$, which could be a result of the $\mathrm{Mg}$ influence in determining the global $\alpha$-element abundance by ASPCAP. For the other $\alpha$-element chemical planes, the separation appears to be largest in $\mathrm{O}$, weaker in $\mathrm{Si}$, and weakest in $\mathrm{Ca}$.

While abundance uncertainties may help obscure differences in $\alpha$-element abundance trends between the $\mathrm{LMg}$ and $\mathrm{HMg}$ populations, the typical measurement uncertainties for each of the $\alpha$-elements are quite similar (at a given metallicity). Thus the larger separation in lighter $\alpha$-elements than heavier ones seems to be from an astrophysical source rather than due to differences in random uncertainties (although systematic errors could still obscure differences). The size of the separation of the $\mathrm{LMg}$ and $\mathrm{HMg}$ populations in different $\alpha$-elements likely arises from the influence of different types of supernovae. For example, the LMg population may have experienced enrichment from a higher fraction Type Ia supernovae. Tsujimoto et al. (1995) have shown that, while Type Ia supernovae have 

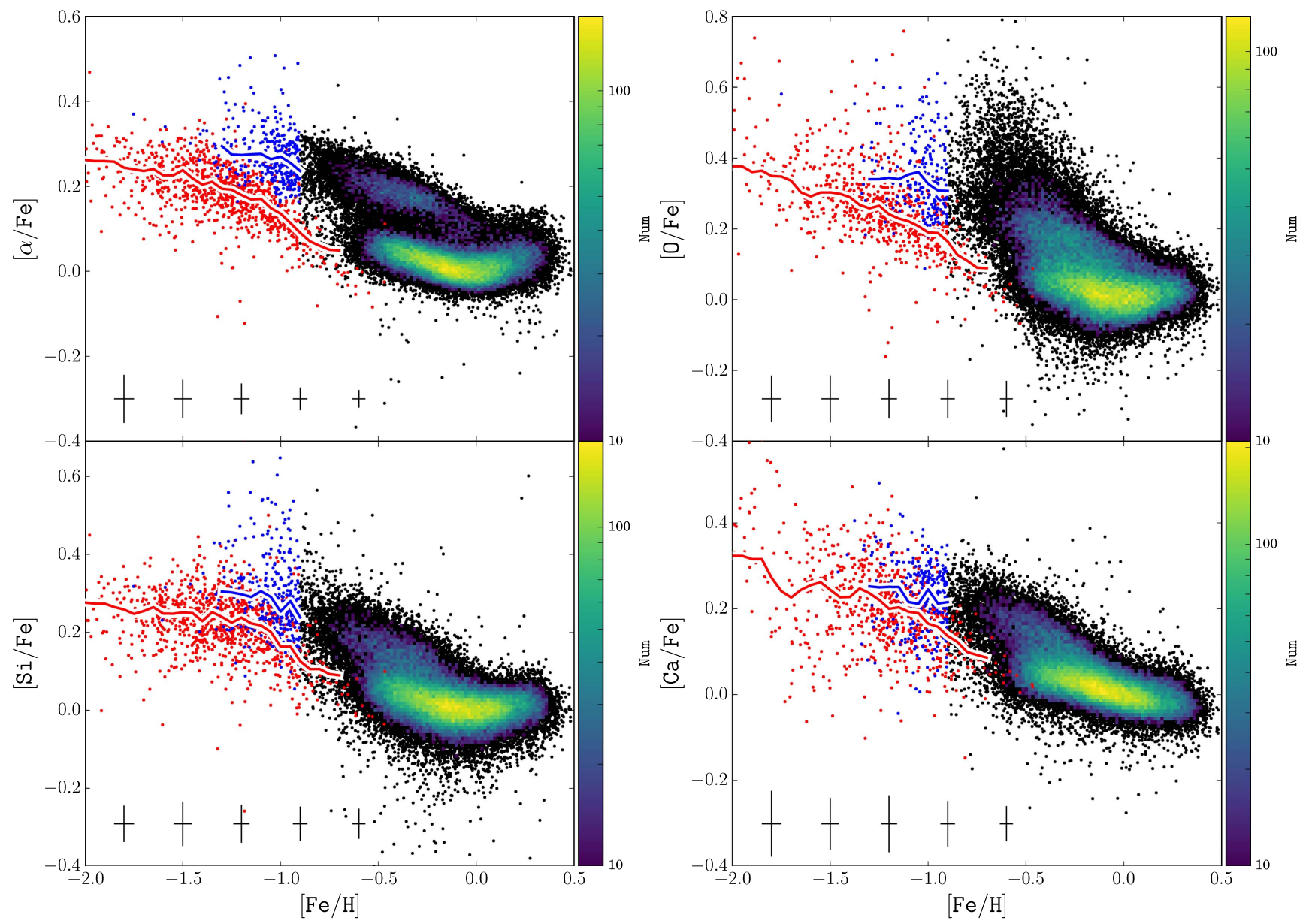

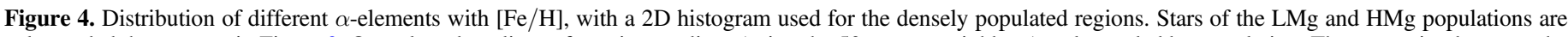

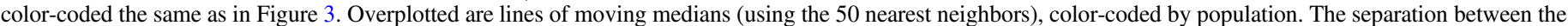

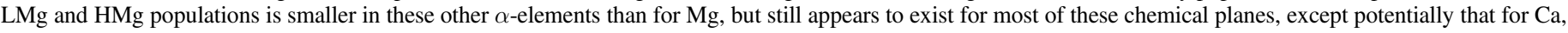
where the metal-poor population overlap is greatest.

contributed a negligible amount of $\mathrm{O}$ and $\mathrm{Mg}$ (about $1 \%$ each) in the solar neighborhood, they have contributed a larger fraction of $\mathrm{Si}(17 \%), \mathrm{Ca}(25 \%)$, and $\mathrm{Fe}(57 \%)$. They show that these fractions increase in lower mass/metallicity systems, such as the LMC, in which Type Ia supernovae still contribute a small fraction of $\mathrm{O}$ and $\mathrm{Mg}(3 \%)$, but make up an even larger fraction of the $\mathrm{Ca}(44 \%)$ and $\mathrm{Fe}(76 \%)$. Another possible explanation for the different separations in $\alpha$-elements is that the two metal-poor populations could have been chemically enriched by supernovae of considerably different progenitor masses or metallicities, which may effect their chemical abundance patterns (see Nomoto et al. 2013).

\subsubsection{Light and Odd-Z Elements: $C, N, A l$, and $K$}

Figure 5 presents some of the light and odd- $Z$ element patterns, and demonstrates a very distinct separation in aluminum at the level of almost $\sim 0.5$ dex. The population with lower $\mathrm{Mg}$ (the $\mathrm{LMg}$ population) is found to be Al-poor, and the higher $\mathrm{Mg}$ population (the $\mathrm{HMg}$ population) has solar or slightly enriched levels of $\mathrm{Al}$, which is consistent with the results from the smaller more metal-rich sample from Hawkins et al. (2015). The gap in Al between the two populations is remarkably large. While it is tempting to use $\mathrm{Al}$ as the primary discriminating element for low metallicity populations, we refrain from doing so now for two reasons: (1) the typically larger ASPCAP uncertainties on $[\mathrm{Al} / \mathrm{Fe}]$ ratios, and (2) much smaller sample sizes when selecting stars based on $\mathrm{Al}$ abundances rather than $\mathrm{Mg}$ abundances because fewer stars have the low uncertainties necessary to make fine chemical distinctions. However, future studies with better aluminum data may find great power in using this element as a discriminator of these two metal-poor populations.

While there is significantly more scatter, we also see some distinction between these two populations in carbon, with the $\mathrm{LMg}$ stars typically having lower $[\mathrm{C} / \mathrm{Fe}]$ (for a given metallicity), although first dredge-up and subsequent mixing for stars at the red giant branch (RGB) bump will bring up $\mathrm{CNO}$-cycle processed material, typically decreasing the surface $[\mathrm{C} / \mathrm{N}]$ from its natal level through a decrease in ${ }^{12} \mathrm{C}$ and an increase in ${ }^{14} \mathrm{~N}$ (Gratton et al. 2000; Karakas \& Lattanzio 2014). In the metallicity range where our two populations overlap, we find that their typical $[\mathrm{N} / \mathrm{Fe}]$ ratios are quite similar. At lower metallicities, the $\mathrm{N}$-abundances increase in the $\mathrm{LMg}$ stars; however, because these are measured from $\mathrm{CN}$ features in APOGEE spectra, which are weak and can disappear for very metal-poor stars (Holtzman et al. 2015), these [N/Fe] ratios are very uncertain (and instead many are upper limits). Note that unlike other chemical abundances from APOGEE, which are calibrated to remove abundance trends with temperature seen in 


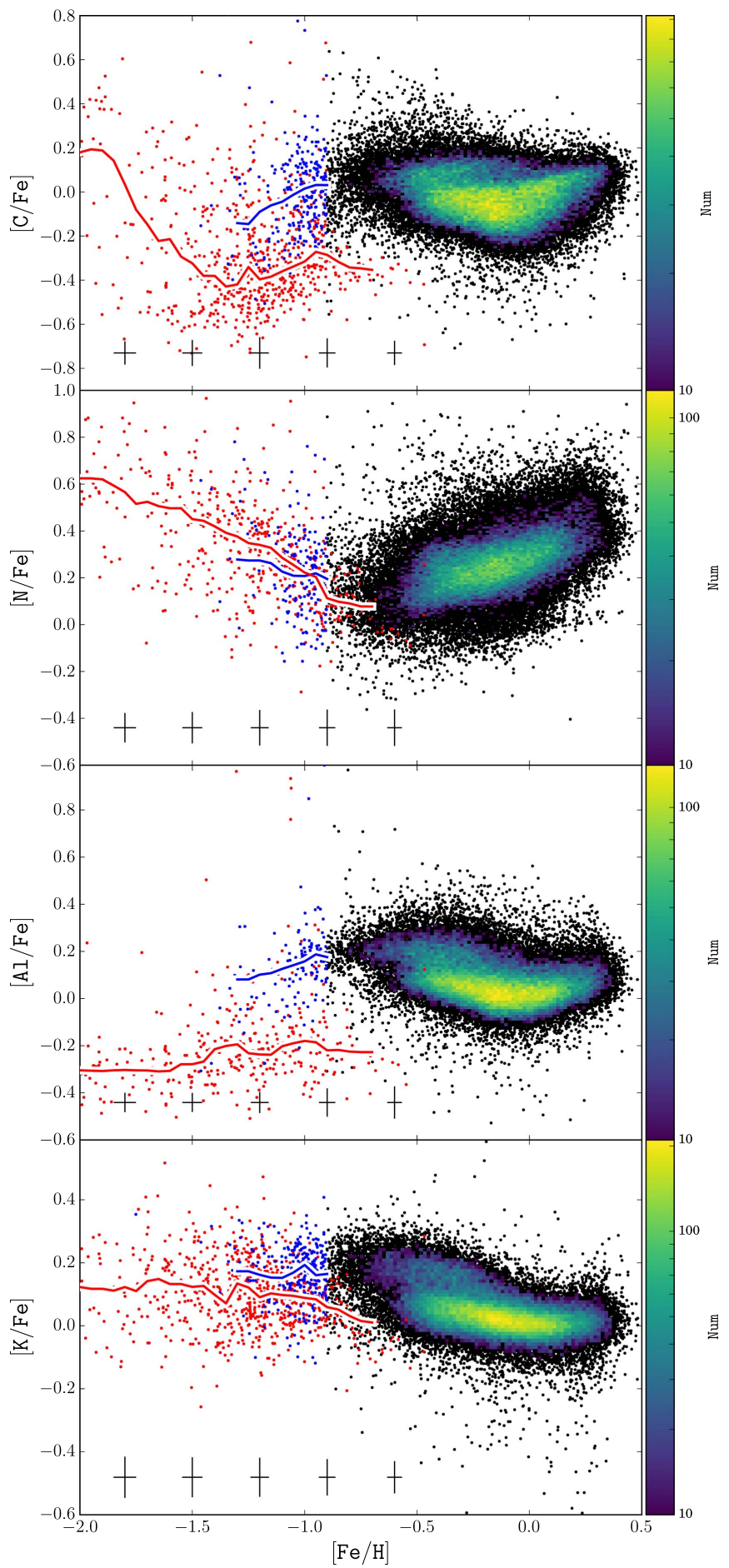

Figure 5. Same as Figure 4, but for the abundance distributions of the light and odd- $Z$ elements $\mathrm{C}, \mathrm{N}, \mathrm{Al}$, and $\mathrm{K}$. The element here for which the two metalpoor populations stand out most distinctly from one another is $\mathrm{Al}$, where the $\mathrm{LMg}$ population appears $\mathrm{Al}$-poor and the $\mathrm{HMg}$ population has approximately solar Al levels.

cluster stars (Holtzman et al. 2015), C and $\mathrm{N}$ are not calibrated in this way because dredge-up and mixing in giants intrinsically produces trends with temperature. The data exhibit only a slight difference in the median $[\mathrm{K} / \mathrm{Fe}]$ ratios between the $\mathrm{LMg}$ and $\mathrm{HMg}$ populations, which is diluted by the large scatter in both populations, such that their $[\mathrm{K} / \mathrm{Fe}]$ distributions appear similar.

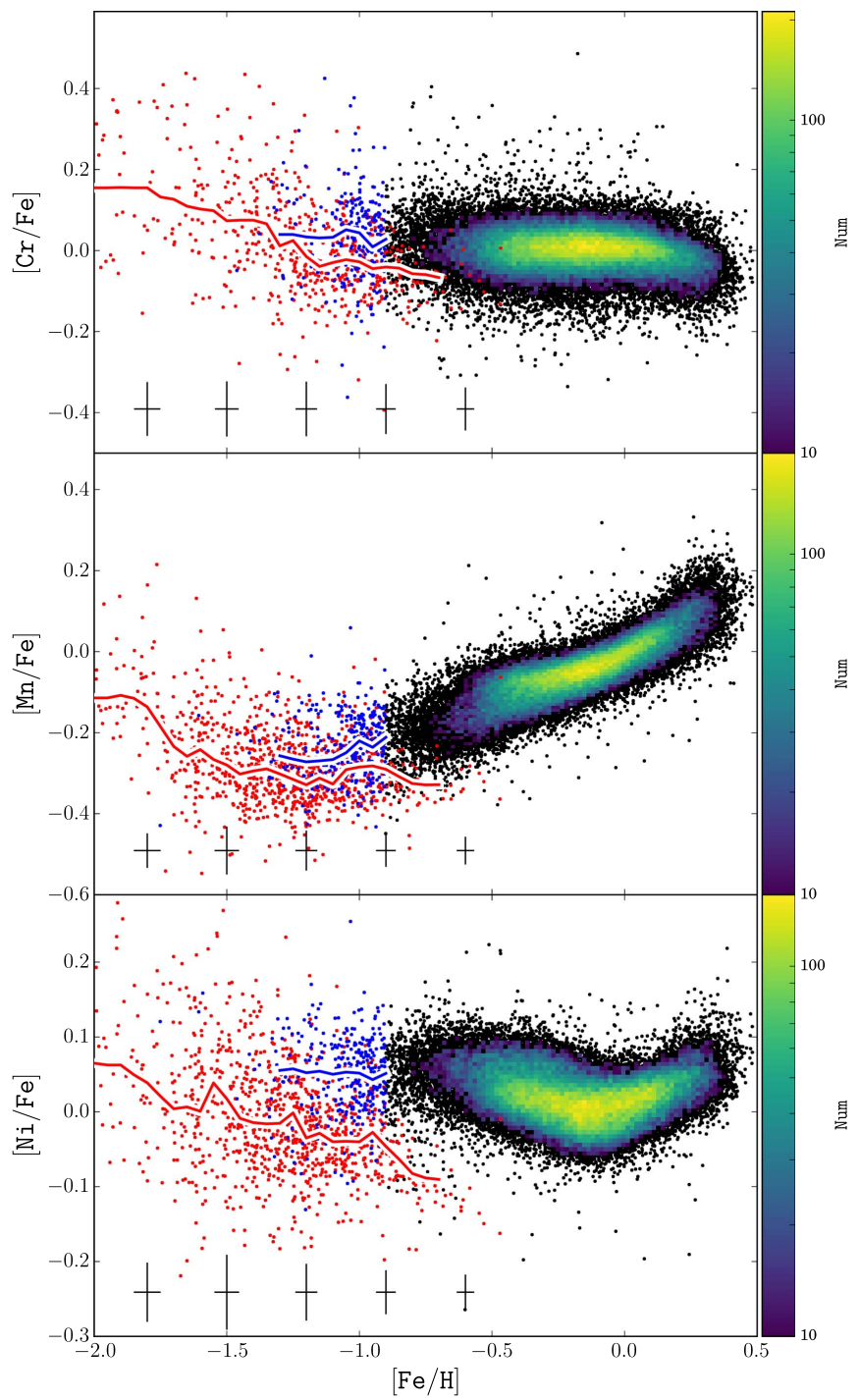

Figure 6. Same as Figure 4, but for the abundance distributions of the ironpeak elements $\mathrm{Cr}, \mathrm{Mn}$, and $\mathrm{Ni}$. Here we see some significant separation of the $\mathrm{LMg}$ and $\mathrm{HMg}$ populations in $\mathrm{Ni}$, in a fashion similar to that seen in some of the $\alpha$-elements.

\subsubsection{Iron Peak Elements: $\mathrm{Cr}, \mathrm{Mn}$, and $\mathrm{Ni}$}

Figure 6 presents the distributions of heavier, iron-peak elements. The most significant separation between these two populations is in $\mathrm{Ni}$, which, although less pronounced than for $\mathrm{Al}$, is similar in appearance to some of the $\alpha$-element abundance separations (as shown in Figure 4). For Ni, we see a slight overlap of the two populations, but most HMg stars have higher $\mathrm{Ni}$ abundances than the $\mathrm{LMg}$ stars, which is again consistent with the findings of Hawkins et al. (2015). Like K, the distributions of $\mathrm{Cr}$ and $\mathrm{Mn}$ abundances for the two populations mostly overlap, although the median of these distributions show slight differences, with the $\mathrm{LMg}$ population having lower $[\mathrm{Cr} / \mathrm{Fe}]$ and $[\mathrm{Mn} / \mathrm{Fe}]$ ratios. The $\mathrm{Cr}$ and $\mathrm{Mn}$ distributions appear to be flat or decreasing with decreasing metallicity above $[\mathrm{Fe} / \mathrm{H}] \gtrsim-1.4$, but at lower metallicities the $\mathrm{LMg}$ population appears to begin increasing in $\mathrm{Cr}$ and $\mathrm{Mn}$ with decreasing metallicity.

For $\mathrm{Cr}$ and $\mathrm{Mn}$, as well as for $\mathrm{Ni}$ and possibly $\mathrm{C}$, at low metallicities there is a slight increase in $[\mathrm{X} / \mathrm{Fe}]$ ratios with decreasing metallicity. The likely reason for this trend is that, at 

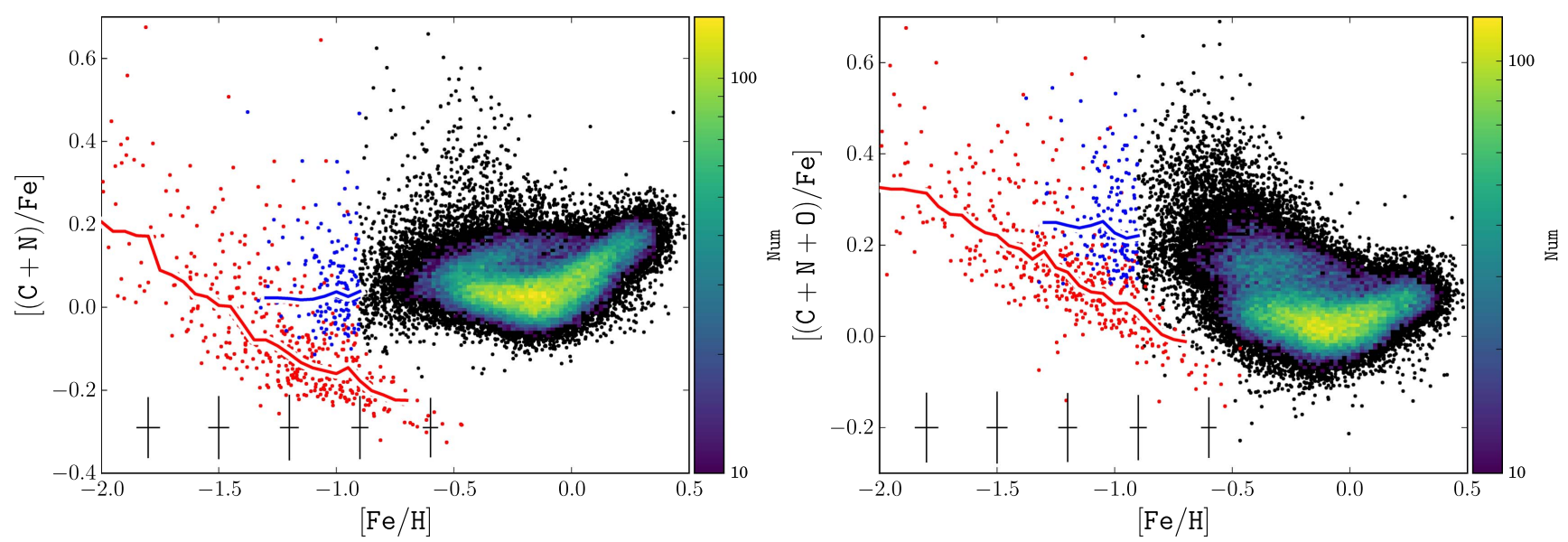

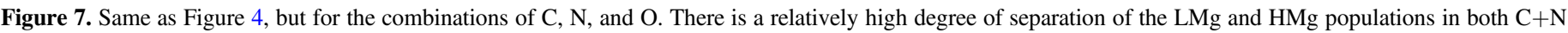

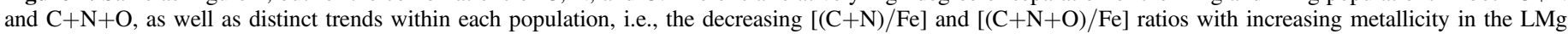
population, and nearly constant ratios in the $\mathrm{HMg}$ population.

low metallicities, the lines used to measure these elements become increasingly weaker, so much so that they should be undetectable at the typical $\mathrm{S} / \mathrm{N}$ of our selection criteria. Thus, the measurements presented at the lowest metallicities would instead be upper limits. We would then expect to see an increasing $[\mathrm{X} / \mathrm{Fe}]$ trend with decreasing metallicity because of two effects. (1) When measuring upper limits, ASPCAP is effectively fitting the noise present in spectra, so for a set of stars with a range in temperature, we expect the derived upper limits to be temperature dependent. Hotter stars have intrinsically weaker lines, so when fitting the same noise level, higher abundances will be derived for these stars than for cool stars, which should have stronger lines for a given abundance. This has the effect of artificially increasing the median abundance ratio at low metallicities where these upper limits appear. (2) We also expect lower abundance ratios to have higher reported uncertainties, and thus to be more likely to result in a star being cut out at these low metallicities by our maximal uncertainty criterion. By tending to remove stars with lower and less certain abundances, we artificially drive up the median abundance ratios at the lowest metallicities (as we approach $[\mathrm{Fe} / \mathrm{H}] \sim-2.0$ ), and we expect this to especially affect elements like $\mathrm{Cr}, \mathrm{Mn}, \mathrm{Ni}$, and possibly $\mathrm{C}$.

These effects should primarily impact $\mathrm{Cr}$, which has the weakest lines of the four elements mentioned above, the abundances reported are more likely to represent upper limits below metallicities of around $[\mathrm{Fe} / \mathrm{H}] \sim-1.5$ or $-1.6 . \mathrm{Cr}$ would then be followed by $\mathrm{Mn}$ and $\mathrm{Ni}$, which would both return upper limits at even lower metallicities. On the other hand, for carbon, it would be more surprising if overestimated line strengths are responsible for the up turn at low metallicities, because there are so many carbon features throughout the APOGEE spectra that are used to derive $[\mathrm{C} / \mathrm{Fe}]$. More of the $[\mathrm{C} / \mathrm{Fe}]$ ratio measurements, therefore, may be real even at lower metallicities. Moreover, stars with high $[\mathrm{C} / \mathrm{Fe}]$ ratios are not unexpected given the existence of carbon-enhanced metal-poor (CEMP) stars with $[\mathrm{C} / \mathrm{Fe}]>+1.0$ (see Beers \& Christlieb 2005; Frebel \& Norris 2015, and references therein), so the trends seen in $[\mathrm{C} / \mathrm{Fe}]$ may well be real.

\subsubsection{Combined Light Elements: $(C+N)$ and $(C+N+O)$}

As mentioned above, first dredge-up and mixing at the RGB bump can effect the surface abundances of (primarily) carbon and nitrogen (with small changes possible for oxygen; Gratton et al. 2000; Karakas \& Lattanzio 2014; Martig et al. 2016), so that these abundances no longer reflect their natal values. However, the total abundance of carbon, nitrogen, and oxygen (as represented by $[(\mathrm{C}+\mathrm{N}+\mathrm{O}) / \mathrm{Fe}])$ should remain unchanged by the dredge-up, and since the birth oxygen abundance is nearly conserved in low-mass stars, the surface $[(\mathrm{C}+\mathrm{N}) / \mathrm{Fe}]$ is also essentially unchanged (Gratton et al. 2000; Martig et al. 2016). Figure 7 shows that most of the stars in the $\mathrm{LMg}$ and $\mathrm{HMg}$ populations exhibit different $\mathrm{C}+\mathrm{N}$ and $\mathrm{C}+\mathrm{N}+\mathrm{O}$ abundances, and they are quite distinct. In both of these abundances, the $\mathrm{HMg}$ exhibits a scatter around solar $[(\mathrm{C}+\mathrm{N}) / \mathrm{Fe}]$ and $[(\mathrm{C}+\mathrm{N}+\mathrm{O}) / \mathrm{Fe}]$ of $+0.2 \mathrm{dex}$. For the same metallicity, the $\mathrm{LMg}$ shows lower $\mathrm{C}+\mathrm{N}$ and $\mathrm{C}+\mathrm{N}+\mathrm{O}$ abundances than the $\mathrm{HMg}$, which provides another example of a chemical space where the $\mathrm{LMg}$ and $\mathrm{HMg}$ populations appear to separate reasonably.

The $\mathrm{LMg}$ stars exhibit decreasing $\mathrm{C}+\mathrm{N}$ and $\mathrm{C}+\mathrm{N}+\mathrm{O}$ abundances with increasing metallicity in addition to having a decreasing scatter with increasing metallicity. The higher scatter at lower metallicities is most likely due to less reliable abundance measurements from weaker lines (primarily poor $\mathrm{N}$ abundances from weak $\mathrm{CN}$ lines) in metal-poor stars, but the concentration in $\mathrm{LMg}$ abundance ratios especially narrows for $[\mathrm{Fe} / \mathrm{H}] \gtrsim-1.3$. This tight trend then continues to metallicities higher than our initial examination cutoff at $[\mathrm{Fe} / \mathrm{H}]=-0.9$, and we can see a $(\mathrm{C}+\mathrm{N})$-poor group of "LMg-extension" stars that reaches to $[\mathrm{Fe} / \mathrm{H}] \sim-0.5$. These stars appear to follow the chemical abundance pattern set by the metal-poor $\mathrm{LMg}$ stars, but have $[(\mathrm{C}+\mathrm{N}) / \mathrm{Fe}]$ (and to some extent even $[(\mathrm{C}+\mathrm{N}$ $+\mathrm{O}) / \mathrm{Fe}])$ ratios that deviate significantly from the canonical thin and thick disk populations, which have $[(\mathrm{C}+\mathrm{N}) / \mathrm{Fe}]$ ratios nearly at or greater than solar. Therefore, we assign these stars to the $\mathrm{LMg}$ population. We do so explicitly by examining the $[\mathrm{Fe} / \mathrm{H}]>-0.9$ stars with subsolar $[(\mathrm{C}+\mathrm{N}) / \mathrm{Fe}]$ and assigning a conservative, by-eye linear expression for the upper $[(\mathrm{C}+\mathrm{N}) / \mathrm{Fe}]$ envelope of this distribution (given numerically by $[(\mathrm{C}+\mathrm{N}) / \mathrm{Fe}]=-0.4[\mathrm{Fe} / \mathrm{H}]-0.46)$. We then define stars with $[(\mathrm{C}+\mathrm{N}) / \mathrm{Fe}]$ under this envelope as potential $\mathrm{LMg}$ stars, but note that this is a conservative selection, again to avoid thin disk contamination. These more metal-rich LMg stars are also shown in previous figures, where 


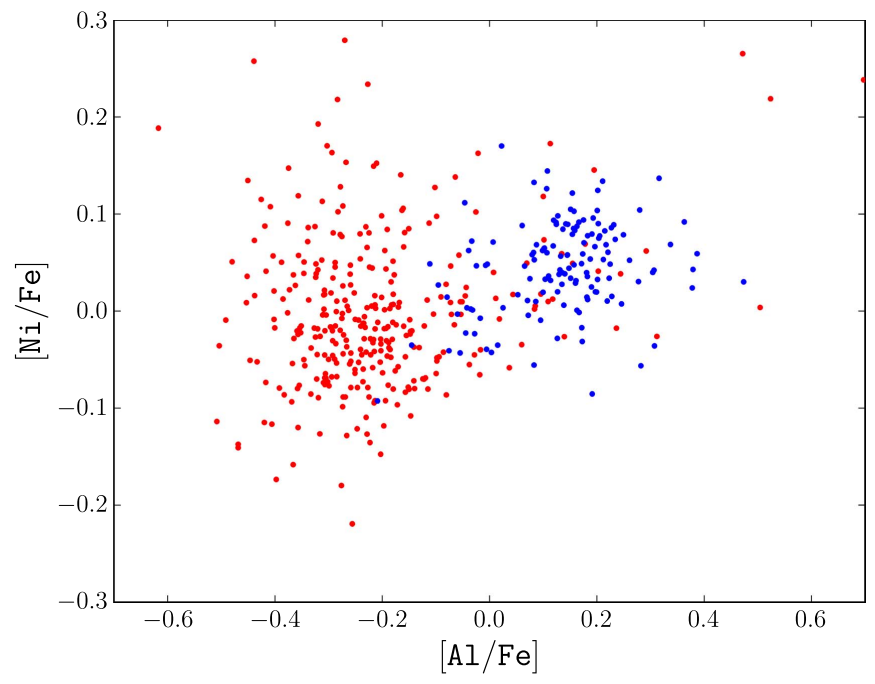

Figure 8. $[\mathrm{Ni} / \mathrm{Fe}]$ vs. $[\mathrm{Al} / \mathrm{Fe}]$ for metal-poor stars $([\mathrm{Fe} / \mathrm{H}]<-0.9)$ in the $\mathrm{LMg}$ (red) and HMg (blue) populations shown in Figure 3. This slice of chemical space is one example where these two populations cluster with good separation, and demonstrate how incorporating different chemical information provides opportunities for further refining the definitions of populations.

they appear to follow other $\mathrm{LMg}$ population trends, and support that these stars are members of this population.

\subsection{Exploring Multi-dimensional Chemical Space}

As demonstrated, the two metal-poor populations we have identified via their $[\mathrm{Mg} / \mathrm{Fe}]$ distributions in the APOGEE database are also quite well discriminated in other elemental ratios, such as $[\mathrm{Al} / \mathrm{Fe}]$ and $[(\mathrm{C}+\mathrm{N}) / \mathrm{Fe}]$. While we have examined stellar abundances of different elements one by one, these stars live in a highly multi-dimensional chemical space that can be sliced in many different ways to search for distinct stellar populations. For example, the $[\mathrm{Ni} / \mathrm{Fe}]$ versus $[\mathrm{Al} / \mathrm{Fe}]-$ plane for stars attributed to the $\mathrm{LMg}$ and $\mathrm{HMg}$ populations in Figure 8, shows a striking separation. This is similar to the separation reported by Nissen \& Schuster (2010), who examined $[\mathrm{Ni} / \mathrm{Fe}]$ versus $[\mathrm{Na} / \mathrm{Fe}]$ for stars between $[\mathrm{Fe} / \mathrm{H}]=-1.6$ and -0.4 , and found two populations (which they also selected based on the $[\mathrm{Mg} / \mathrm{Fe}]-[\mathrm{Fe} / \mathrm{H}]$ ) like those examined here. This perhaps should be expected, because $\mathrm{Na}$ and $\mathrm{Al}$ are produced through the $\mathrm{NeNa}$ and $\mathrm{MgAl}$ cycles, which are linked and operate under similar temperature ranges (Arnould et al. 1999); thus $\mathrm{Na}$ and $\mathrm{Al}$ abundances should be roughly correlated.

But again, Figure 8, like all preceding figures are just twodimensional slices through chemical space, when we have many more dimensions that we can utilize simultaneously. While it is difficult to visualize higher dimensionalities, we can use tools such as clustering algorithms to search this space to provide statistically rigorous tests of our proposed separations.

To conduct such a multi-dimensional probe and to quantify how well the two populations and their differences are captured by our simple selection in $[\mathrm{Mg} / \mathrm{Fe}]$ versus $[\mathrm{Fe} / \mathrm{H}]$, we utilize two clustering algorithms to independently and objectively look for these populations. The multi-dimensional space we search is that of metallicity $([\mathrm{Fe} / \mathrm{H}]),[(\mathrm{C}+\mathrm{N}) / \mathrm{Fe}]$ (which should be more representative of birth abundances than $\mathrm{C}$ or $\mathrm{N}$ separately due to the effects of first dredge-up, as discussed earlier), and $[\mathrm{X} / \mathrm{Fe}]$ for $\mathrm{O}, \mathrm{Mg}, \mathrm{Al}, \mathrm{Si}, \mathrm{K}, \mathrm{Ca}, \mathrm{Cr}, \mathrm{Mn}$, and $\mathrm{Ni}$,

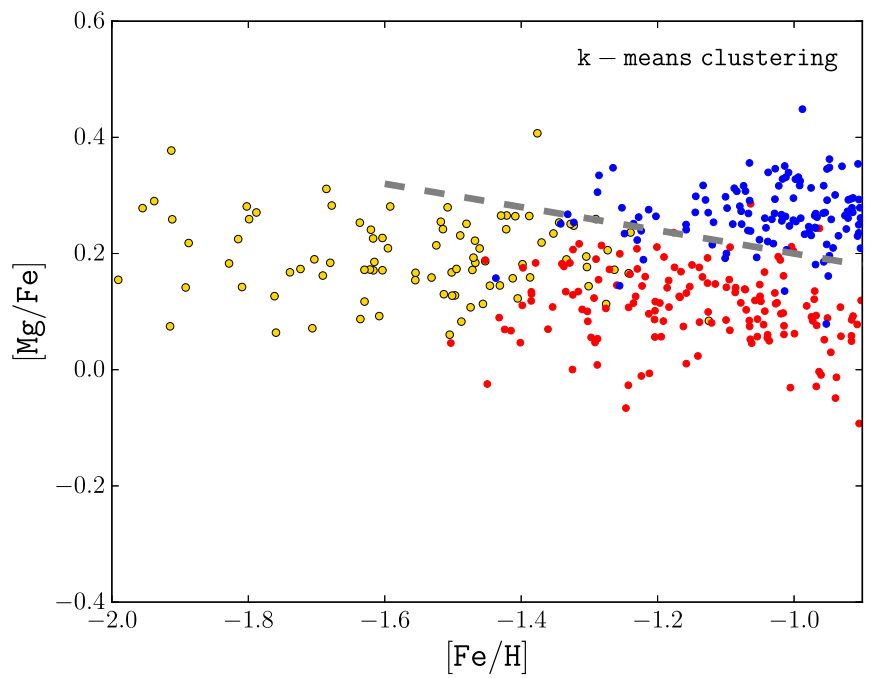

Figure 9. $[\mathrm{Mg} / \mathrm{Fe}]$ vs. $[\mathrm{Fe} / \mathrm{H}]$ projection of the $11 \mathrm{D}$ chemical space probed, where we have performed clustering analyses on stars with well defined chemical abundances, as described in the text. Stars are color-coded by cluster assignment according to the k-means clustering algorithm, two of which (colored red and blue) are very similar to the two populations that we defined by eye in Figure 3.

i.e., those elements with good data that were previously examined.

First, we use an algorithm to perform k-means clustering (MacQueen 1967) to search for clusters in the aforementioned 11-dimensional chemical space for all stars with uncertainties under $0.1 \mathrm{dex}$ and $[\mathrm{Fe} / \mathrm{H}] \leqslant-0.9$ (so that the populations noted in this work are not lost to the much more populous thin and thick disk chemical distributions). We performed a silhouette analysis (Rousseeuw 1987) to determine the optimal number of clusters $(k)$ to represent the data, finding that three clusters best describe the data. The resulting assignment of stars for the three clusters are shown in the $[\mathrm{Mg} / \mathrm{Fe}]-[\mathrm{Fe} / \mathrm{H}]$ plane in the left panel of Figure 9 color-coded according to their cluster assignment by the k-means algorithm.

Of the three clusters identified, two seem to separate primarily in metallicity from the third cluster of stars typically having $[\mathrm{Fe} / \mathrm{H}] \lesssim-1.4$ and entirely representing the lowest metallicity stars. This third cluster may reflect the fact that at metallicities below $[\mathrm{Fe} / \mathrm{H}] \sim-1.5$ the $\mathrm{LMg}$ and $\mathrm{HMg}$ populations blend together into one chemically indistinguishable group. Alternatively this third group may be a spurious bifurcation of one of the other two clusters (presumably the cluster corresponding to the $\mathrm{LMg}$ ), either as an artifact of the k-means algorithm or due to low statistics creating a small gap in an otherwise continuous sequence. Whether there may be an astrophysical reason for this distinct, metal-poor population should be reconsidered if it persists despite more data or improved techniques applied to this problem.

The remaining two k-means clusters are located at higher metallicities, where the $\mathrm{LMg}$ and $\mathrm{HMg}$ are more distinct. How these clusters relate to the populations defined by our visual inspection of only the two-dimensional $[\mathrm{Mg} / \mathrm{Fe}]-[\mathrm{Fe} / \mathrm{H}]$ plane is shown by the overplotted dividing line we initially used to separate the $\mathrm{LMg}$ and $\mathrm{HMg}$ populations in Figures 2 and 3. As may be seen, our adopted dividing line appears to properly separate most of the stars assigned to either of the more metalrich clusters defined independently by the k-means algorithm. 
Apart from the most metal-poor cluster, we note that the k-means clustering has produced one relatively low- and one relatively high-Mg cluster. Specifically, we find that of the stars below and above the line in Figure 9, respectively, 90\% (146/163) of the LMg population stars are assigned to the low-Mg k-means cluster and 95\% (103/108) of the HMg stars are assigned to the high-Mg k-means cluster. Thus, the k-means algorithm identifies clusters relatively consistent (at least at the higher metallicity range of our sample) with the two populations we specified using our by-eye division based on only two chemical dimensions. This suggests that $[\mathrm{Mg} / \mathrm{Fe}]$ and metallicity alone are a robust discriminator of the two groups of relatively metal-poor stars. We also note that most of the crosscontamination occurs around our dividing line, and somewhat at the metal-poor end of the high- $\mathrm{Mg}$ or $\mathrm{HMg}$ population distribution, where the third, metal-poor cluster dominates.

The other clustering algorithm that we try is DBSCAN (Ester et al. 1996), a two parameter density-based clustering algorithm that builds clusters by chaining together data points that have a minimum of $N$ neighbors within a multi-dimensional sphere of radius $\epsilon$. Together, these parameters determine a minimum "density" and the algorithm identifies clusters present in the data with that density. Because this algorithm is density based, it will tend to exclude data on the outskirts of clusters, and can be fairly sensitive to the choice of input parameters (which effectively define the desired densities of output clusters). Nevertheless, DBSCAN also delivers results consistent with our by-eye selection in finding two clusters (with input parameters of $N=17$ and $\epsilon=0.21 \mathrm{dex}$ ).

Of the stars assigned to the two DBSCAN clusters, 94\% (119/127) of the low Mg abundance cluster stars would be properly associated with the $\mathrm{LMg}$ population according to the by-eye definition, and $91 \%(64 / 70)$ of the stars assigned to the high-Mg abundance cluster would be identified as $\mathrm{HMg}$ population stars. The remaining 163 stars lie in less densely populated regions of chemical space than the cores of the clusters and are thus unassigned to either of these clusters. Because of this, as was the case with the k-means clustering analysis, the two clusters found by DBSCAN that seem to correspond to the $\mathrm{LMg}$ and $\mathrm{HMg}$ populations are predominately populated at the higher metallicities of this sample $([\mathrm{Fe} / \mathrm{H}] \gtrsim-1.5)$, leaving the lower metallicity stars unassigned. While our adopted values of the DBSCAN $N$ and $\epsilon$ parameters are not definitive (and indeed alternative pairings produce similar clusters, such as those shown in Figure 9), DBSCAN clustering analysis reveals that there is a density threshold that produces two distinct clusters with a manner of separation that is consistent with our initial separation in the $[\mathrm{Mg} / \mathrm{Fe}]-[\mathrm{Fe} / \mathrm{H}]$ plane.

In summary, we find that the k-means and DBSCAN algorithms reaffirm our by-eye discrimination, and identify very similar clusters to those we identify as the $\mathrm{LMg}$ and $\mathrm{HMg}$ populations at the metallicities where we see the largest differences in chemical distributions. This is an objective affirmation that these populations are real, and that our method to separate them in a single projection of the $[\mathrm{Mg} / \mathrm{Fe}]-[\mathrm{Fe} / \mathrm{H}]$ plane properly assigns $90 \%$ or more of stars to the correct population as compared to the results of clustering algorithms. While, for simplicity, we proceed with the use of a strict twodimensional, Mg-based division of the two metal-poor populations, there will naturally be a small degree of crosscontamination (as we saw with the comparison to the k-means and DBSCAN results), due to some intrinsic overlap of these populations, the projection of a multi-dimensional distribution into two dimensions, and uncertainties blurring the intrinsic distribution of these populations. In the future, when truly large samples of multi-dimensional data are available for metal-poor stars, purer discrimination will be possible by looking at multiple chemical dimensions.

\subsection{Kinematical Nature of the LMg and HMg Populations}

While these two populations appear chemically distinct, they would be even more astronomically significant if they additionally exhibit different kinematics, which we can examine using the radial velocities of stars measured by APOGEE. We convert these radial velocity into the Galactic Standard of Rest system assuming a solar motion of $\left(V_{r}, V_{\phi}, V_{z}\right)_{\odot}=(14,250,7) \mathrm{km} \mathrm{s}^{-1}$ (Schönrich et al. 2010; Schönrich 2012). Majewski et al. (2012) have shown the utility of the Galactic longitude- $V_{\mathrm{GSR}} / \cos (b)$ diagram for revealing stellar populations kinematically using only radial velocity data. $V_{\mathrm{GSR}} / \cos (b)$ is a proxy for the planar velocity of a star projected onto our line of sight, but breaks down at high Galactic latitudes (Majewski et al. 2012), so in this examination we only use stars with $|b|<62^{\circ}$ (to include the stars in the APOGEE fields centered at $b=60^{\circ}$ ).

Figure 10 shows that the distribution of $V_{\mathrm{GSR}} / \cos (b)$ versus Galactic longitude for the $\mathrm{LMg}$ population has a large velocity dispersion (roughly $150-200 \mathrm{~km} \mathrm{~s}^{-1}$, drawn from Figure 10) with very little to no net rotation, typical of that expected for a halo population. The $\mathrm{HMg}$ population, on the other hand, has a modest velocity dispersion of about $80-120 \mathrm{~km} \mathrm{~s}^{-1}$ around a significant trend of net rotation at the level of about $120-150 \mathrm{~km} \mathrm{~s}^{-1}$ (taken from the amplitude of the sinusoidal velocity variation displayed in ther right panel of Figure 10); the latter is consistent with the rotational velocity for the thick disk, at least at lower metallicities (Chiba \& Beers 2000; Lee et al. 2011; Adibekyan et al. 2013; Allende Prieto et al. 2016). This is perhaps unsurprising, since chemically, the $\mathrm{HMg}$ population looks like an extension of the thick disk. Included in the $\mathrm{HMg}$ population are a few stars that have radial velocities more typical of halo-like kinematics, which may be halo stars with chemistry similar to the thick disk, or contamination from the $\mathrm{LMg}$ population.

Because the $\mathrm{LMg}$ population spans a wider range in metallicity than the $\mathrm{HMg}$ population and one would expect more stellar contribution from the halo (rather than the disk) toward lower metallicities, it is of interest to confirm that the above kinematical signatures persist even at the higher metallicity end of our samples. To do so, we examine $V_{\mathrm{GSR}} / \cos (b)$ versus Galactic longitude only for stars with metallicities $[\mathrm{Fe} / \mathrm{H}]>-1.1$ in each of these populations (Figure 11). Acknowledging the much smaller net samples, we still find that even the more metal-rich stars of the $\mathrm{LMg}$ exhibit halo-like motions, which further justifies that the $\mathrm{LMg}$ population is a coherent and distinct population from the dynamically colder $\mathrm{HMg}$ population.

\section{Discussion}

\subsection{Relation to High- $\alpha$ and Low- $\alpha$ Halo Stars}

Our analysis of the APOGEE database has focused on a very specific examination of a large sample of metal-poor stars making use of the clear separation seen in the $[\mathrm{Mg} / \mathrm{Fe}]-[\mathrm{Fe} / \mathrm{H}]$ 


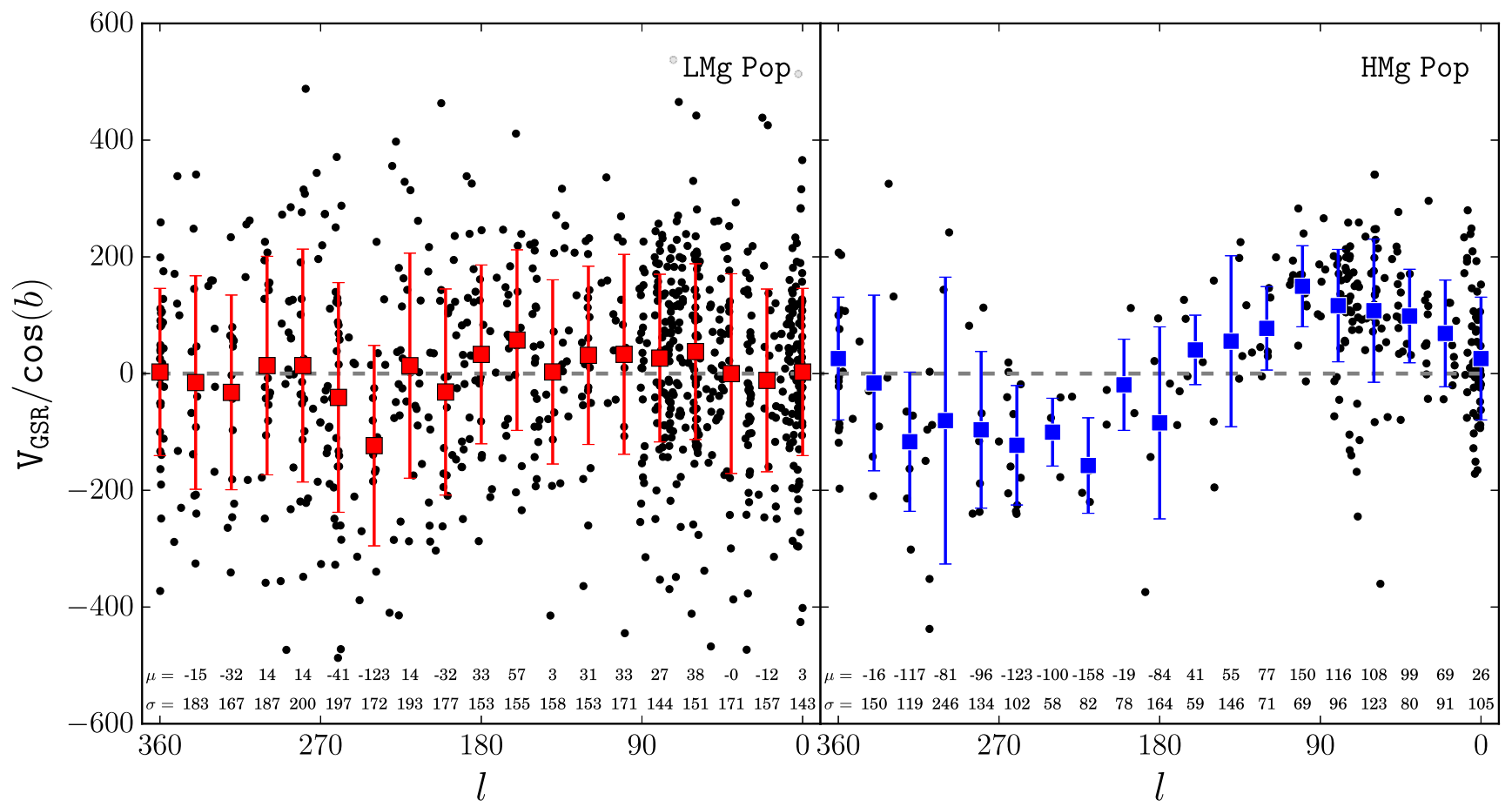

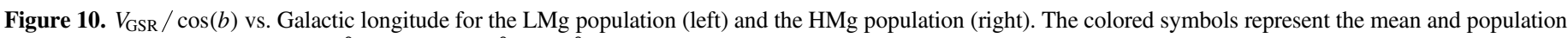

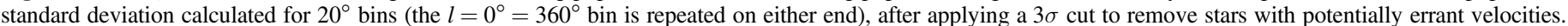

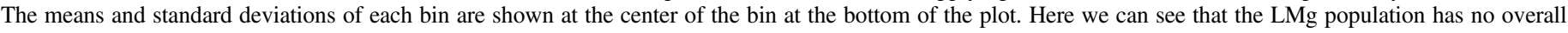

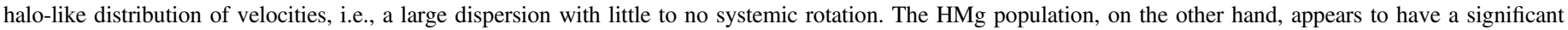
rotation with a much smaller dispersion.

plane, a separation also validated in other chemical dimensions, like $[\mathrm{Al} / \mathrm{Fe}]-[\mathrm{Fe} / \mathrm{H}]$ and $[(\mathrm{C}+\mathrm{N}) / \mathrm{Fe}]-[\mathrm{Fe} / \mathrm{H}]$, as well as in the overall 11D chemical space (see Section 3.3). Previous studies of smaller samples of stars have demonstrated a split of halo stars into high- and low- $\alpha$ groups (Nissen \& Schuster 2010, 2011; Navarro et al. 2011; Ramírez et al. 2012; Schuster et al. 2012; Sheffield et al. 2012; Jackson-Jones et al. 2014; Hawkins et al. 2015). These groups appear generally to correspond well with our $\mathrm{HMg}$ and $\mathrm{LMg}$ populations, as we now demonstrate.

In a study of the abundances of $\alpha$-elements $(\mathrm{Mg}, \mathrm{Si}, \mathrm{Ca}, \mathrm{Ti})$, $\mathrm{Na}, \mathrm{Cr}$, and $\mathrm{Ni}$ for 94 kinematically and metallicity selected dwarf stars, Nissen \& Schuster (2010) found two populations of stars with halo-like kinematics (total space velocities, $V_{\text {tot }}>180 \mathrm{~km} \mathrm{~s}^{-1}$ ) separated in the $[\mathrm{Mg} / \mathrm{Fe}]-[\mathrm{Fe} / \mathrm{H}]$ plane. The population of halo stars with lower $[\mathrm{Mg} / \mathrm{Fe}]$ also separated from the higher $[\mathrm{Mg} / \mathrm{Fe}]$ ratio population in other $\alpha$-elements (although to a lesser extent; Nissen \& Schuster 2010; Ramírez et al. 2012; Hawkins et al. 2015), and other elements, such as $(\mathrm{C}+\mathrm{N}), \mathrm{Na}, \mathrm{Al}, \mathrm{Ni}, \mathrm{Cu}, \mathrm{Zn}, \mathrm{Y}$, and $\mathrm{Ba}$, whereas little to no distinction was seen for some elements such as $\mathrm{Cr}$ and $\mathrm{Mn}$ (Nissen \& Schuster 2010, 2011; Hawkins et al. 2015).

Figure 12 compares the chemistry of the APOGEE DR13 sample of this study to the stellar abundances presented in Nissen \& Schuster (2010, 2011). We find general agreement for most elements, with perhaps small offsets between the two data sets in a few cases. The largest differences may be seen in the distribution of $[\mathrm{Ca} / \mathrm{Fe}]$ ratios of the two populations seen here, which is likely due to the different methods of spectroscopic analysis employed by Nissen \& Schuster (2010, 2011) and APOGEE. Nissen \& Schuster (2010, 2011) measured abundances relative to two bright thick disk stars to achieve a high internal precision, but may be subject to systematic offsets compared to chemical abundance measurements using different methods, such as APOGEE's automated spectroscopic analysis. This difference in method of analysis, along with the use of differing spectral lines, model atmospheres, etc., may lead to the offsets seen in $[\mathrm{Ca} / \mathrm{Fe}]$ ratios as well as those in other elements.

In addition to the chemical similarities between the low- and high- $\alpha$ halo stars and our HMg and LMg populations, there are kinematical similarities linking these groups of stars. Both the thick disk and high- $\alpha$ halo stars have (on average) higher rotational velocities than the low- $\alpha$ halo stars (Nissen \& Schuster 2010), analogous to the kinematical differences seen between the $\mathrm{HMg}$ and the $\mathrm{LMg}$ populations here (see Figure 10). Thus, kinematics affirm that the low- $\alpha$ halo stars are members of the same population as the $\mathrm{LMg}$ population stars identified here, and that the high- $\alpha$ halo stars are part of the $\mathrm{HMg}$ population. While the $\mathrm{LMg} / \mathrm{low}-\alpha$ halo stars and $\mathrm{HMg} /$ high- $\alpha$ halo stars seem to be samples of the same respective populations, we maintain the usage of the names $\mathrm{LMg}$ and HMg to more explicitly reflect their selection through $\mathrm{Mg}$ abundances, the $\alpha$-element that most easily distinguishes these populations.

It is interesting that there is such good agreement between the samples of stars in our work and Nissen \& Schuster (2010, 2011), given the vastly different volumes sampled by each work. APOGEE surveys a large volume, allowing it to reach into the bulge or out into the halo. In contrast, Nissen \& Schuster $(2010,2011)$ studied a sample of stars from the solar neighborhood, extending only as far as $\sim 335 \mathrm{pc}$. The fact that both studies find similar distributions of stars suggests that they come from parent populations that, in terms of their distribution, do not vary significantly with position in the Galaxy. By concluding that the populations studied here are the 


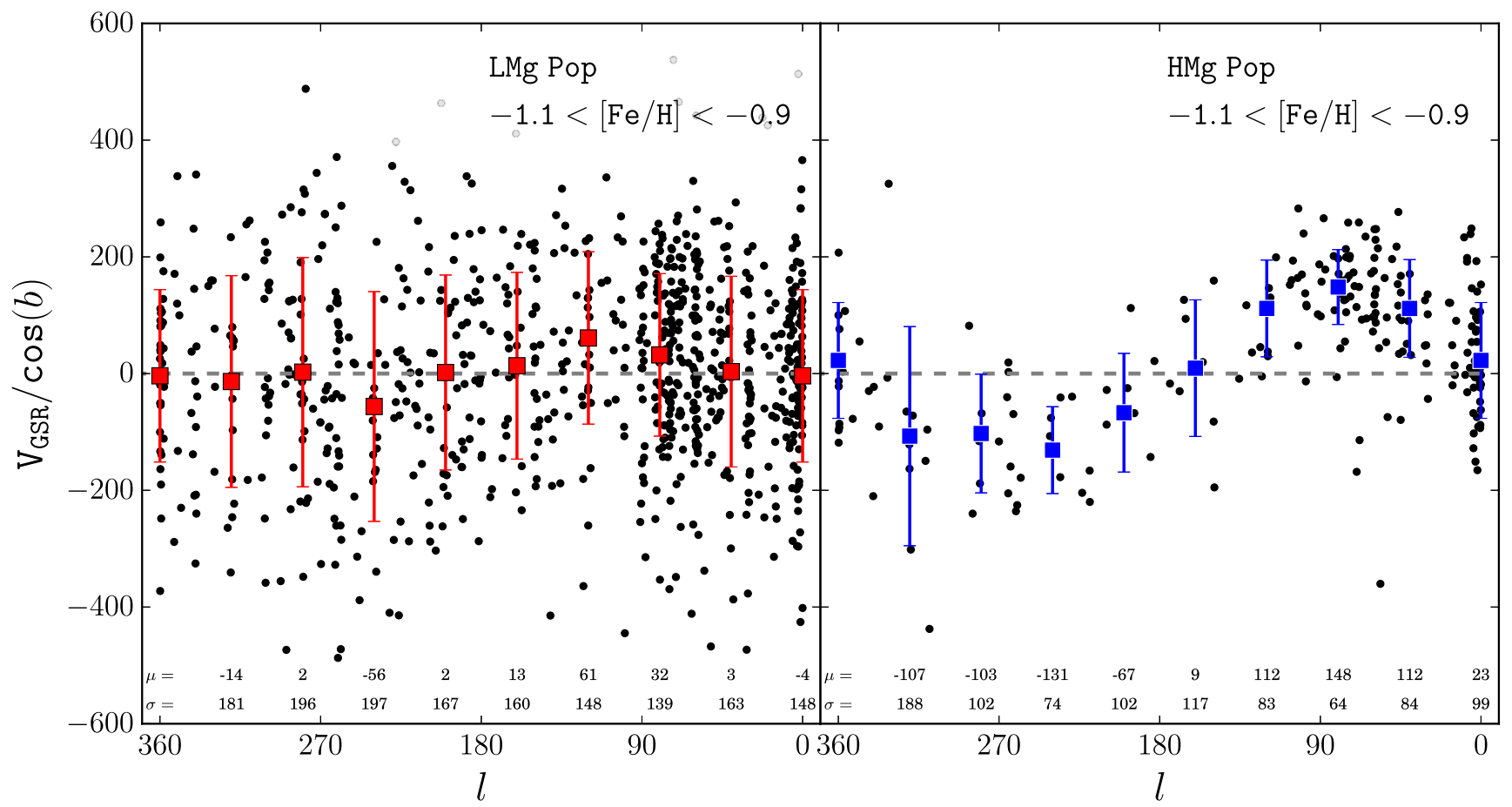

Figure 11. Same as Figure 10, with data binned into $40^{\circ}$ bins, for $\mathrm{LMg}$ and $\mathrm{HMg}$ stars with $-1.1<[\mathrm{Fe} / \mathrm{H}]<-0.9$. The more metal-rich ends of these populations follow the same kinematical trends as the subsamples covering the larger metallicity range shown in Figure 10.

same or related to those revealed by the high- and low- $\alpha$ halo stars (initially seen by Nissen \& Schuster 2010), APOGEE uses its large statistical sampling to bring more clarity and significance to these two distinct populations.

\subsection{Comparison to MW Satellites}

One possible origin for metal-poor stars in the MW is through the accretion of smaller, dwarf spheroidal (dSph) systems. As noted in the past (Venn et al. 2004; Tolstoy et al. 2009), dSph stars typically have lower $\alpha$-element abundances than most MW stars at the same metallicities. However, at lower metallicities $([\mathrm{Fe} / \mathrm{H}] \lesssim-1.5)$, there is more overlap in $[\mathrm{X} / \mathrm{Fe}]$ between the chemistry of $\mathrm{MW}$ and dSph stars. This suggests that, at least at higher metallicities, dSph stars from lower mass dwarf galaxies, like those common around the MW, are unlikely to contribute significantly to either the $\mathrm{LMg}$ or HMg populations. This does not, however, rule out the possibility that satellite galaxies could have contributed stars to our halo with different chemistry or that $\mathrm{dSphs}$ could have contributed stars at lower metallicities where the agreement is better.

The sample of dSph stars examined in some of these past studies come from multiple dSph galaxies. While this gives us an idea of the general spread of abundances across $\mathrm{dSph}$ satellites, it does not provide a picture of the chemical evolution within a given satellite. If we want to assess the $\mathrm{dSph}$ populations that are more likely to have been contributed to the MW, we should focus on the chemical evolution in more massive satellites, because previous studies (Bullock \& Johnston 2005; Font et al. 2006) have found that satellites accreted earlier in the history of a galaxy are expected to be, on average, more massive. This is likely to have an impact on the chemistry of these satellites, because we might expect more massive satellites to have experienced more enrichment before Type Ia supernovae began to contribute their yields to the interstellar medium (e.g., due to higher star formation rates, higher star formation efficiency, better retention of supernovae products, etc.). This would have the effect of pushing the $[\alpha / \mathrm{Fe}]-\mathrm{knee}$ of these satellites to higher metallicities leading to potentially higher $[\alpha / \mathrm{Fe}]$ ratios than less massive satellites for a given metallicity, and resulting in better agreement with the metal-poor stars seen in the MW at a given metallicity.

Although a few of these more massive satellites were somewhat represented in past studies, we wish to compare the APOGEE sample to a larger set of abundances from one of them - the Fornax dSph-by examining the red giant abundances measured from high-resolution spectra by Letarte et al. (2010) and Lemasle et al. (2014). We also compare our APOGEE sample to the chemical abundances of Large Magellanic Cloud (LMC) red giants derived from highresolution spectra by Van der Swaelmen et al. (2013) and of Sagittarius (Sgr) dSph and M54 stars measured from mediumresolution spectra by Mucciarelli et al. (2017). We show the $\mathrm{Mg}$ and $\mathrm{Ca}$ abundance distributions for each of these systems in comparison to our APOGEE sample in Figure 13. These two chemical elements show trends where chemical abundance pattern differences appear to show up most distinguished either among MW populations or between satellites and the MW.

The top panels of Figure 13 show that Fornax stars exhibit $[\mathrm{Mg} / \mathrm{Fe}]$ ratios on the low side of the $\mathrm{LMg}$ population's chemical abundance pattern, except at the lowest metallicities where the agreement is better. On the other hand, the Fornax $\mathrm{Ca}$ abundances do not agree well with most of the stars observed by APOGEE and instead $[\mathrm{Ca} / \mathrm{Fe}]$ ratios of Fornax stars are on average lower than those of both the $\mathrm{LMg}$ and $\mathrm{HMg}$ populations at all metallicities. In the distribution of heavy elements, we find that the differences between Fornax and LMg stars in Ni abundances are similar to that in $\mathrm{Mg}([\mathrm{Ni} / \mathrm{Fe}]$ is slightly lower in Fornax by about a tenth of a dex on average), whereas their $\mathrm{Cr}$ abundance distributions 

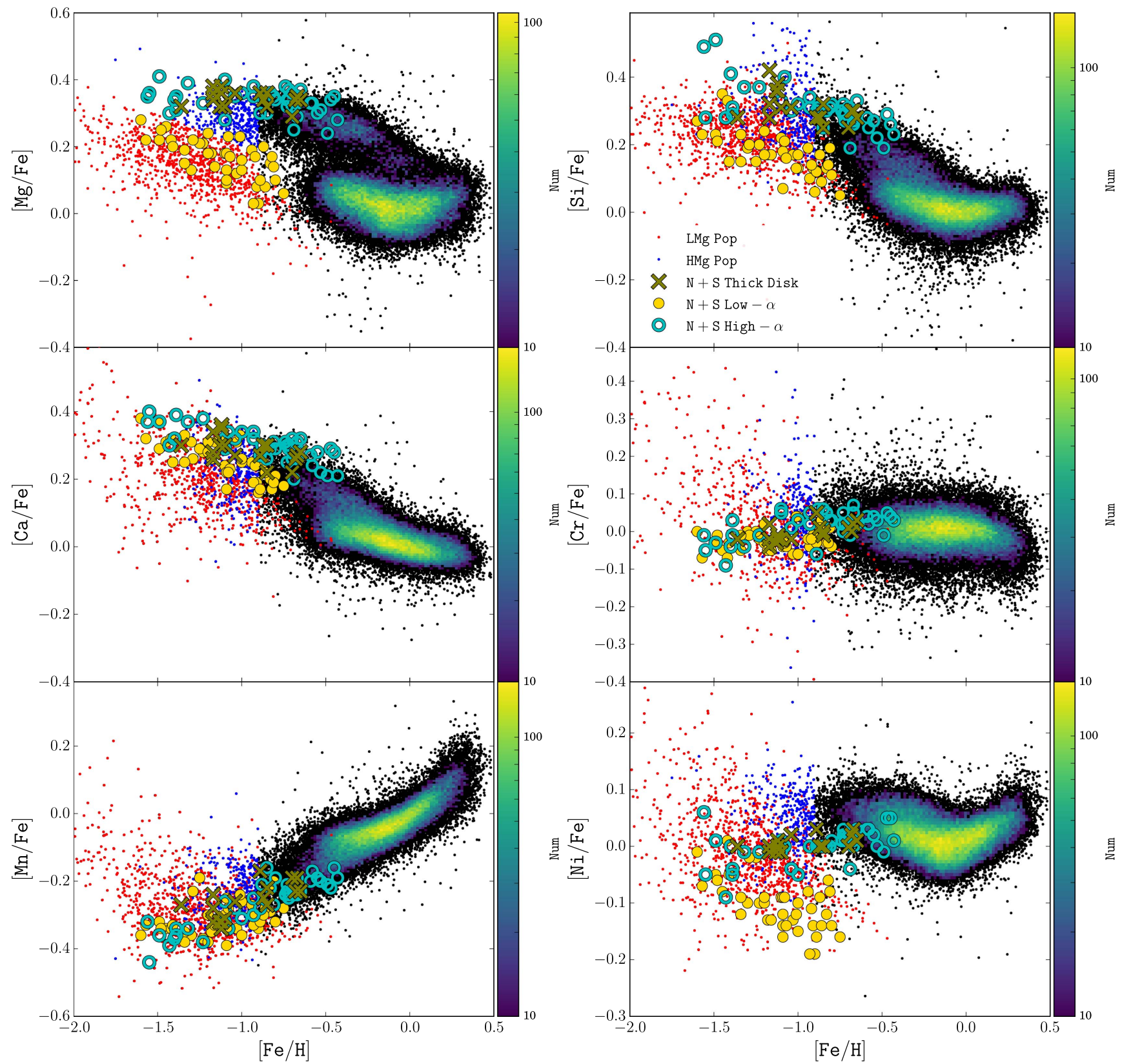

Figure 12. Distribution of $[\mathrm{X} / \mathrm{Fe}]$ with $[\mathrm{Fe} / \mathrm{H}]$ for $\mathrm{Mg}, \mathrm{Si}, \mathrm{Ca}, \mathrm{Cr}, \mathrm{Mn}$, and $\mathrm{Ni}$ with a $2 \mathrm{D}$ histogram of the densely distributed stars as done in Figure 1 . Stars of $\mathrm{LMg}$ and $\mathrm{HMg}$ populations are color-coded the same as in Figure 3. Overplotted are data from Nissen \& Schuster (2010, 2011) color-coded to identify kinematically selected thick disk stars (olive green crosses), and their chemically selected high- $\alpha$ (cyan open circles) and low- $\alpha$ (yellow filled circles) halo stars.

differ more significantly, like $\mathrm{Ca}([\mathrm{Cr} / \mathrm{Fe}]$ is lower in Fornax by a couple tenths of a dex on average).

In contrast to Fornax, giants from the more massive LMC (shown in Figure 13) typically have higher $[\mathrm{X} / \mathrm{Fe}]$ ratios. At metallicities $[\mathrm{Fe} / \mathrm{H}] \lesssim-1.0$, there is better agreement between the LMC giants and our LMg stars among their $\alpha$-element and Fe-peak abundances, e.g., the distributions of $[\mathrm{Mg} / \mathrm{Fe}]$ and $[\mathrm{Ca} / \mathrm{Fe}]$ ratios shown in the middle panels of Figure 13. This may suggest that the metal-poor stars in our $\mathrm{LMg}$ population and the LMC have had analogous star formation histories, and ones that differ from both lower mass dSph satellites, and some of the more massive dSphs, such as Fornax. At higher metallicities $[\mathrm{Fe} / \mathrm{H}] \gtrsim-1.0$, the $\mathrm{LMC}$ giants look like a chemical extension of the $\mathrm{LMg}$ stars.
Unfortunately, one of the most massive dSphs and therefore interesting satellites to compare with our Mg populations, the Sgr dSph, has been observed by APOGEE, but has been mostly excluded from our own sample by the stellar surface temperature restriction $T_{\text {eff }}>4000 \mathrm{~K}$. So that we maintain as self-consistent a sample as possible, the latter requirement removes the coolest and brightest red giants from our sample, which have been analyzed by ASPCAP using a different grid of model atmospheres. These infrared-bright stars, however, are the only types of red giants that APOGEE has accessed and have data available to analyze in Sgr (e.g., Majewski et al. 2013; Hasselquist et al. 2017) because of the large distances to this $\mathrm{dSph}$. For the same reason, but also because these younger stars are the dominant red giant population in the system, most 

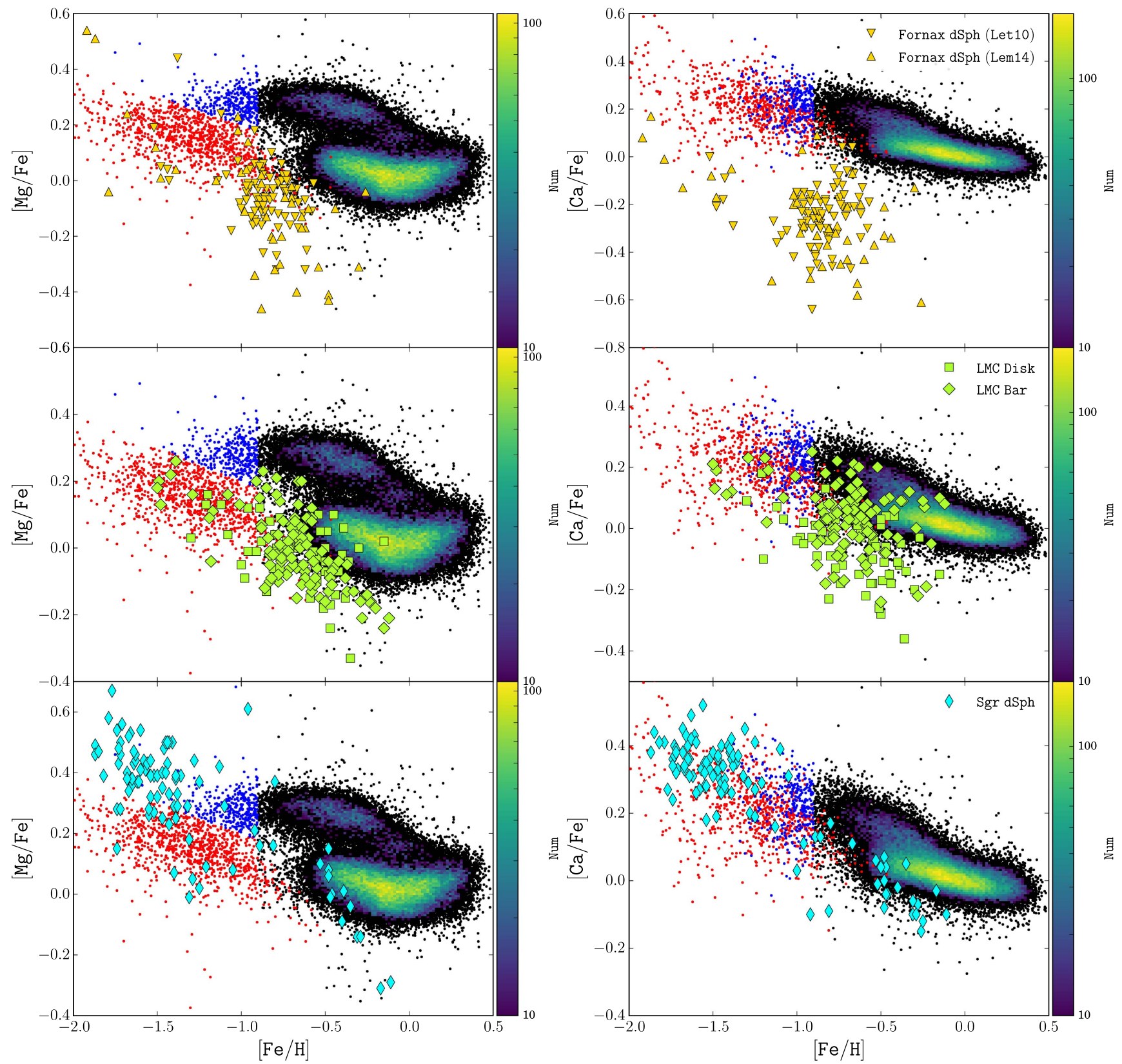

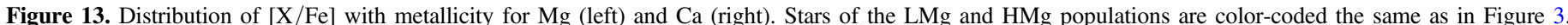

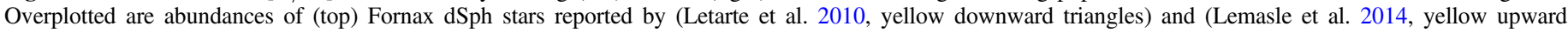

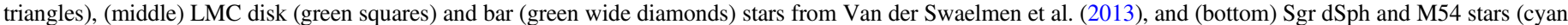
narrow diamonds) reported by Mucciarelli et al. (2017).

other chemical abundance studies of the Sgr dSph also typically observe Sgr's more metal-rich stars (e.g., Sbordone et al. 2007; Hasselquist et al. 2017). Nevertheless, while this younger, more metal-rich Sgr dSph population is not directly comparable to our more metal-poor populations, it has been noted to resemble a chemical extension of the low- $\alpha$ metalpoor stars in the MW (Hasselquist et al. 2017) - i.e., our LMg population.

A new study of the Sgr dSph, by Mucciarelli et al. (2017) appears to bear out this suggestion. Using abundances of $\alpha$-elements measured from medium-resolution spectra, these authors show that both Sgr dSph and M54 stars (located at the center of the $\mathrm{Sgr} \mathrm{dSph}$ ) have similar $\alpha$-element chemical abundance patterns to LMC stars. As may be seen in the bottom panels of Figure 13, and as is the case of LMC stars, there is an overlap in $\mathrm{Mg}$ and $\mathrm{Ca}$ abundances of the Mucciarelli et al. (2017) Sgr dSph and M54 stars with the LMg population (and to a smaller extent, the HMg population).

\subsection{Potential Origins}

As discussed above, the present dSph satellites of the MW typically have $\alpha$-element abundances that are too low to explain the origin of most MW stars observed by APOGEE (and even the halo stars shown in Venn et al. 2004; Tolstoy et al. 2009), at metallicities $[\mathrm{Fe} / \mathrm{H}] \gtrsim-1.5$, where we are interested in exploring the origin of the $\mathrm{LMg}$ and $\mathrm{HMg}$ populations. This is demonstrated in past studies and in our comparison with Fornax in Figure 13. The one possible 
exception is the $\mathrm{Sgr} \mathrm{dSph}$, for which the dominant population looks like a possible metal-rich extension of the $\mathrm{LMg}$ population (Sbordone et al. 2007; Carretta et al. 2010; Hasselquist et al. 2017).

Even if Sgr dSph stars may look more chemically similar to the $\mathrm{LMg}$ (as is being revealed by larger samples that push to lower metallicities, see Mucciarelli et al. 2017), it seems unlikely that this particular satellite could have contributed the majority of the LMg stars. This is evidenced by the full sky coverage of the $\mathrm{LMg}$ population with halo-like kinematics, whereas the Sgr dSph and its tidal tails are confined roughly to a plane in the sky (Majewski et al. 2003; Law \& Majewski 2010). Thus the majority of the LMg population (and HMg population, which has still higher $\alpha$-element abundances) does not seem to be accounted for by the accretion of $\mathrm{dSph}$ satellites like most of those around the MW now, particularly at higher metallicities, $[\mathrm{Fe} / \mathrm{H}] \gtrsim-1.5$.

As mentioned above, $\Lambda \mathrm{CDM}$ predictions, however, suggest that galaxies accreted earlier in the history of our Galaxy will tend to be more massive, resulting in higher $[\alpha / \mathrm{Fe}]$ ratios than those being accreted today, for stars of the same metallicity (Font et al. 2006; Lee et al. 2015). Additionally, cosmological hydrodynamical simulations including chemical evolution have reported complex scenarios where some of the accreted satellites could continue star formation activity in a bursty mode, producing stellar populations with a variety of levels of $\alpha$-element enrichment (e.g., Font et al. 2006; Tissera et al. 2012). The variation in the assembly histories of MW-mass galaxies has been shown theoretically to then shape the chemical patterns of their stellar halos (Font et al. 2006; Tissera et al. 2013).

Fernández-Alvar et al. (2017a), using APOGEE data combined with distances, found that the innermost regions of the Galactic halo are dominated by stars with higher $[\alpha / \mathrm{Fe}]$ ratios, but that dominance shifts to stars with lower $[\alpha / \mathrm{Fe}]$ ratios at larger distances, at least for the moderately metal-poor regime probed by APOGEE (i.e., stars with $[\mathrm{Fe} / \mathrm{H}] \gtrsim-2$ ). This $[\alpha / \mathrm{Fe}]$ variation supports the idea that more massive satellites with faster enrichment or star formation, and thus higher $[\alpha / \mathrm{Fe}]$ ratios, may have been accreted earlier in the history of the MW to help form the inner regions of the halo.

The lower $[\alpha / \mathrm{Fe}]$ ratios of our $\mathrm{LMg}$ population compared to the metal-poor end of the thick disk, yet higher $[\alpha / \mathrm{Fe}]$ ratios than current MW dSph satellites may then be evidence that these stars have been accreted from more massive dwarf systems early in the history of the MW. Alternatively, the LMg population may have originated from regions in the early MW halo with star formation similar to what would be expected in more massive dwarf galaxies. It is interesting that the $\mathrm{LMg}$ population, a potentially accreted population, is a significant fraction of the metal-poor stars observed by APOGEE, at least between metallicities of about -1.5 and -0.9 .

Fishlock et al. (2017) recently presented a study that examined neutron capture element abundances in stars selected from Nissen \& Schuster (2010). They found that in terms of light and heavy s-process elements ( $l s$ and $h s$ respectively) the low- $\alpha$ halo stars have higher $[h s / l s]$, which affirms results from Nissen \& Schuster (2011), who found similar differences in $[\mathrm{Ba} / \mathrm{Y}]$ (Ba is an $h s$ - and $\mathrm{Y}$ an $l s$-element). Fishlock et al. (2017) also found differences between these two populations in terms of their ratios of $\mathrm{Y}$ to $\mathrm{Eu}$ (an r-process element) and ratios of other s-process elements to $\mathrm{Eu}$. This is significant because the low $[\mathrm{Y} / \mathrm{Eu}]$ ratios exhibited by the low- $\alpha$ halo stars, along with high $[\mathrm{Ba} / \mathrm{Y}]$ ratios, are signatures seen in $\mathrm{dSph}$ stars, so that these neutron capture element patterns further support the accretion origin for the equivalent of our $\mathrm{LMg}$ population.

These conclusions are in agreement with those that have been drawn for the origin of low- $\alpha$ halo stars (Nissen \& Schuster 2010; Sheffield et al. 2012; Hawkins et al. 2015), with which the LMg population seems to be associated. In addition to exhibiting lower abundances of $\alpha$ and other elements (Nissen \& Schuster 2010, 2011; Sheffield et al. 2012; Hawkins et al. 2015), low- $\alpha$ halo stars have been shown to have ages typically 2-3 Gyr younger than high- $\alpha$ halo and thick disk stars at any given metallicity, as well as larger orbital radii and distances from the Galactic mid-plane (Schuster et al. 2012). Additionally, Schuster et al. (2012) found that the low- $\alpha$ halo stars they observed had larger eccentricities, clumped at values greater than 0.85 (i.e., $0.85 \lesssim e \lesssim 1.0$ ), whereas the observed high- $\alpha$ halo stars exhibit a greater spread in eccentricities $(0.4 \lesssim e \lesssim 1.0)$. The results of these and various other studies lend further support to the hypothesis that the low- $\alpha$ halo stars have been accreted.

In contrast to the likely accretion origin for the $\mathrm{LMg}$, the $\mathrm{HMg}$ population's apparent net rotation and chemical similarity to the thick disk suggest an in situ formation similar or related to that of the thick disk. If so, the $\mathrm{HMg}$ population might simply be a metal-poor extension of the thick disk, and the two may share an origin, whether through (1) dissipative collapse (Majewski 1993), (2) radial migration (Sellwood \& Binney 2002, note, however, that several recent simulations suggest that radial migration does not sufficiently heat the disk of the Galaxy—see Minchev et al. 2012; Vera-Ciro et al. 2014), or (3) by being "kicked out" or heated from initially colder orbits (possibly in the bulge or the colder disk) into more halo-like orbits by multibody encounters or the accretion of satellites (possibly even those that contributed the accreted halo stars; Quinn et al. 1993; Walker et al. 1996; Nissen \& Schuster 2010; Schuster et al. 2012; Sheffield et al. 2012; Johnston 2016).

Another possibility, proposed by Hawkins et al. (2015), based on the chemical similarities between high- $\alpha$ halo stars and the thick disk, is that there may be a smooth transition between what they call the "canonical halo" and the thick disk, both of which they suggest formed in situ. The $\mathrm{HMg}$ population might then represent an intermediate, transitional stage between these two populations. Because the $\mathrm{HMg}$ population has chemical abundance patterns similar to the thick disk, but with a lower apparent net rotation than the thick disk, it may be related to the MWTD reported by Chiba \& Beers (2000) and Beers et al. (2002). If these two populations are the same, or are related, this would further support an in situ formation of the $\mathrm{HMg}$ population, as was proffered as the potential origin of the MWTD.

The bifurcation of properties in metal-poor stars is reminiscent of the classic bimodality in Horizontal Branch (HB) types of the "Younger Halo" and "Old Halo" globular clusters between metalliticities $-1.8<[\mathrm{Fe} / \mathrm{H}]<-0.8$, which were thought to have been accreted from satellites and formed in situ respectively (Zinn 1993, 1996) ${ }^{23}$ While these globular cluster populations are no longer thought to be distinct in age

\footnotetext{
23 At lower metallicities, $[\mathrm{Fe} / \mathrm{H}]<-1.8$, Zinn (1996) identifies a third group of metal-poor globular clusters that are spatially and kinematically distinct from the other two globular cluster populations, similar to the three-part division we found in metal-poor stars with the k-means clustering algorithm (Section 3.3).
} 
alone (due to complications in the differences between HB types; Gratton et al. 2010), recent studies have found that there are two distinct age-metallicity relations among globular clusters (Leaman et al. 2013; VandenBerg et al. 2013; Wagner-Kaiser et al. 2017) that cover similar age ranges, with the more metal-poor branch being about $2 \mathrm{Gyr}$ younger than the metal-rich branch for a given metallicity. In this paradigm, the more metal-poor and distant clusters are thought to have been accreted, whereas the more metal-rich clusters with more disk-like kinematics would have formed in situ. With this picture of dual origins for globular clusters, it appears that both globular clusters and field stars separate consistently into in situ and accreted populations.

Putting the $\mathrm{HMg}$ and $\mathrm{LMg}$ populations within the context of prior studies of the thick disk and halo of the MW would benefit from the addition of full kinematics and spatial information for the APOGEE sample. With the Gaia satellite about to deliver parallaxes and proper motions for stars at these magnitudes, this will soon be a reality.

\section{Conclusions}

We detect evidence for two distinct populations of metalpoor stars observed by APOGEE, discriminated by their $[\mathrm{Mg} / \mathrm{Fe}]$. We study the chemistry and kinematics of these populations, and find multiple differences in their properties. The separation between these populations in $[\mathrm{Mg} / \mathrm{Fe}]$ is more pronounced for metallicities $[\mathrm{Fe} / \mathrm{H}] \gtrsim-1.5$. While these populations are also distinguished by the patterns of other $\alpha$-elements, their distinctiveness is less apparent for heavier $\alpha$-elements such as $\mathrm{Ca}$. This variation in chemical separation may reflect some of the finer details of the differing nucleosynthetic processes forming these two populations such as the differential production and contribution of $\alpha$-elements in Type Ia supernovae or in Type II supernovae of different masses or metallicities (Tsujimoto et al. 1995; Nomoto et al. 2013). In addition to the $\alpha$-element differences, the $\mathrm{LMg}$ and $\mathrm{HMg}$ populations are distinct in their $\mathrm{C}+\mathrm{N}, \mathrm{Al}$, and $\mathrm{Ni}$ abundances relative to $\mathrm{Fe}$. While our selection of the two populations used a by-eye discrimination in $[\mathrm{Mg} / \mathrm{Fe}]-[\mathrm{Fe} / \mathrm{H}]$ space, we have also used two different clustering algorithms to search for distinct groupings in an 11-dimensional APOGEE chemical space. Both of these methods generally reproduce our original selection and identify essentially the same two populations apparent in the $[\mathrm{Mg} / \mathrm{Fe}]-[\mathrm{Fe} / \mathrm{H}]$ plane.

We show that the LMg population exhibits halo-like kinematics, with little rotation and a large velocity dispersion of about $150-200 \mathrm{~km} \mathrm{~s}^{-1}$. The $\mathrm{HMg}$ population appears to be kinematically colder, with a rotational velocity $\sim 120-150 \mathrm{~km} \mathrm{~s}^{-1}$ and smaller velocity dispersion around $80-120 \mathrm{~km} \mathrm{~s}^{-1}$. This HMg population, however, includes some stars with radial velocities more consistent with halo-like orbits, similar to those found in other studies such as Nissen \& Schuster (2010), and may reflect a chemical overlap between the $\mathrm{LMg}$ and $\mathrm{HMg}$ populations or a history tied to the formation of the thick disk (given the similarity between the chemistries of the HMg population and the thick disk).

Previous studies have also reported the detection of $\alpha$ element abundance differences in metal-poor stars, with some making selections specifically in $\mathrm{Mg}$, as performed here (Nissen \& Schuster 2010; Navarro et al. 2011; Ishigaki et al. 2012; Sheffield et al. 2012; Jackson-Jones et al. 2014; Hawkins et al. 2015), albeit with fewer stars. The advantage of our study is that we rely on a large sample of stars that have homogeneously determined abundances for many chemical species. In addition, our sample is much larger in size, even at low metallicities, $[\mathrm{Fe} / \mathrm{H}]<-1.0$, where we have more than 1000 stars, which more than doubles the sample in JacksonJones et al. (2014), the largest of these studies. Our analysis is of a sample that is free from kinematical biases, and probes a larger volume of the MW. Finally, both by visual inspection and through the results of more sophisticated clustering algorithms, we are able to identify and separate the two distinct populations noted in past studies with greater statistical significance and reliability than before.

From the chemistry and kinematics of these two populations, we conclude that our LMg population is likely an accreted population of halo stars, formed in conditions similar to those in early dwarf galaxy satellites. Examining the elemental abundance patterns of dSph stars (from Venn et al. 2004; Letarte et al. 2010; Lemasle et al. 2014), we find that our LMg population stars have generally higher $[\alpha / \mathrm{Fe}]$ ratios for stars with metallicities $[\mathrm{Fe} / \mathrm{H}] \gtrsim-1.5$. Thus it appears that if these stars (at least the more metal-rich LMg stars) were accreted earlier in the history of the MW, they were likely accreted from more massive satellites than present dSphs (Font et al. 2006).

Our HMg population, from its chemistry and its slow but significant net rotation, appears to contain mostly stars in the metal-poor end of the thick disk and/or may be related to the potentially distinct component of the MW, the MWTD (Chiba \& Beers 2000; Beers et al. 2002). Within this population, there are also stars that may have halo-like kinematics but chemistry similar to thick disk stars. This would be consistent with the similarities between the Nissen \& Schuster $(2010,2011)$ thick disk and halo high- $\alpha$ stars, who suggest that the high- $\alpha$ halo stars (or equivalently our HMg stars with halo kinematics) may also be part of the dissipative component that also formed the thick disk. This is similar to the picture presented by Sheffield et al. (2012), who suggested that these stars could be in situ stars formed in such a dissipative collapse, or could be stars from the thick disk that were kicked into halo orbits. The $\mathrm{HMg}$ population may then represent a combination of these possibilities.

Measuring more properties of the stars in these two populations may help us further distinguish them, provide more clues to their origins, and/or identify more subpopulations. The origin of $\mathrm{Eu}$ in the low- $\alpha$ halo stars (LMg population) seen by Fishlock et al. (2017) is still not understood, and its relative abundance to s-process elements cannot be accounted for by the slow enrichment and low-mass (1-3 $\left.M_{\odot}\right)$ AGB pollution Fishlock et al. (2017) use to explain the differences in $l s$ and $h s$ abundances in these stars. Thus, as they suggest, further study of these populations with more r-process elements and larger samples may provide a better picture of the chemical evolution of metal-poor stars.

Expanded three-dimensional velocities would greatly expand our ability to study the kinematics and dynamics of the stars in these populations, but will require proper motions. As suggested by Navarro et al. (2011) and Schuster et al. (2012), the three-dimensional motions of stars in the low- $\alpha$ halo population provide evidence for accretion, so space motions would allow us to perform similar analyses with the populations seen in APOGEE. Additionally, full space motions may help separate populations that have distinct kinematics but similar chemistry. The physical distribution of the MW stars in 
our defined populations will be aided by incorporating accurate distance measurements (an initial study of the distribution of metal-poor stars in APOGEE is given in Fernández-Alvar et al. 2017a). Finally, the companion paper by (FernándezAlvar et al. 2017b, Paper II in this series), further explores the chemical evolution of the two distinct metal-poor $\mathrm{LMg}$ and $\mathrm{HMg}$ populations identified in APOGEE.

The authors would like to thank the anonymous referee for constructive comments and improvements, making this a better paper. This research has made use of the Tool for OPerations on Catalogues And Tables (Taylor 2005). C.R.H. and S.R.M. acknowledge National Science Foundation grants AST1312863 and AST-1616636. C.A.P. is thankful to the Spanish MINECO for funding for this research through the grant AYA2014-56359-P. W.J.S. wishes to thank the PAPIIT of Mxico for financial support through project IN103014. L.C. thanks the financial support provided by CONACyT of México (grant 241732), by PAPIIT of México (IG100115, IA101215, IA101517) and by MINECO of Spain (AYA2015-65205-P). T.C.B. acknowledges partial support from grant PHY 14-30152; Physics Frontier Center/JINA Center for the Evolution of the Elements (JINA-CEE), awarded by the US National Science Foundation. J.G. F.-T., D.G., and B.T. gratefully acknowledge support from the Chilean BASAL Centro de Excelencia en Astrofísica y Tecnologías Afines (CATA) grant PFB-06/2007. D.A.G.H. was funded by the Ramón y Cajal fellowship number RYC-2013-14182. D.A.G. H. and O.Z. acknowledge support provided by the Spanish Ministry of Economy and Competitiveness (MINECO) under grant AYA-2014-58082-P. R.R.L. acknowledges support by the Chilean Ministry of Economy, Development, and Tourism's Millennium Science Initiative through grant IC120009, awarded to The Millennium Institute of Astrophysics (MAS). R.R.L. also acknowledges support from the STFC/Newton Fund ST/M007995/1 and the CONICYT/Newton Fund DPI20140114.

Funding for the Sloan Digital Sky Survey IV has been provided by the Alfred P. Sloan Foundation, the U.S. Department of Energy Office of Science, and the Participating Institutions. SDSS-IV acknowledges support and resources from the Center for High-Performance Computing at the University of Utah. The SDSS website is http://www.sdss.org.

SDSS-IV is managed by the Astrophysical Research Consortium for the Participating Institutions of the SDSS Collaboration including the Brazilian Participation Group, the Carnegie Institution for Science, Carnegie Mellon University, the Chilean Participation Group, the French Participation Group, Harvard-Smithsonian Center for Astrophysics, Instituto de Astrofísica de Canarias, The Johns Hopkins University, Kavli Institute for the Physics and Mathematics of the Universe (IPMU)/University of Tokyo, Lawrence Berkeley National Laboratory, Leibniz Institut für Astrophysik Potsdam (AIP), Max-Planck-Institut für Astronomie (MPIA Heidelberg), Max-Planck-Institut für Astrophysik (MPA Garching), MaxPlanck-Institut für Extraterrestrische Physik (MPE), National Astronomical Observatory of China, New Mexico State University, New York University, University of Notre Dame, Observatário Nacional / MCTI, The Ohio State University, Pennsylvania State University, Shanghai Astronomical Observatory, United Kingdom Participation Group, Universidad Nacional Autónoma de México, University of Arizona,
University of Colorado Boulder, University of Oxford, University of Portsmouth, University of Utah, University of Virginia, University of Washington, University of Wisconsin, Vanderbilt University, and Yale University.

\section{ORCID iDs}

Steven R. Majewski (ib https://orcid.org/0000-00032025-3147

Matthew Shetrone (i) https://orcid.org/0000-0003-0509-2656 Timothy C. Beers (i) https://orcid.org/0000-0003-4573-6233 Ricardo Carrera (ib https://orcid.org/0000-0001-6143-8151 Doug Geisler (iD https://orcid.org/0000-0002-3900-8208 Sara Lucatello (iD https://orcid.org/0000-0001-8808-0073 Allison M. Matthews (iD https://orcid.org/0000-00026479-6242

Dante Minniti (iD https://orcid.org/0000-0002-7064-099X Baitian Tang (iD https://orcid.org/0000-0002-0066-0346 Patricia B. Tissera (iD https://orcid.org/0000-0001-5242-2844

\section{References}

Adibekyan, V. Z., Figueira, P., Santos, N. C., et al. 2013, A\&A, 554, A44 Albareti, F. D., Allende Prieto, C., Almeida, A., et al. 2017, ApJS, 233, 25 Allen, C., Poveda, A., \& Schuster, W. J. 1991, A\&A, 244, 280 Allende Prieto, C., Kawata, D., \& Cropper, M. 2016, A\&A, 596, A98 Arnould, M., Goriely, S., \& Jorissen, A. 1999, A\&A, 347, 572 Beers, T. C., Carollo, D., Ivezić, Ž, et al. 2012, ApJ, 746, 34 Beers, T. C., \& Christlieb, N. 2005, ARA\&A, 43, 531

Beers, T. C., Drilling, J. S., Rossi, S., et al. 2002, AJ, 124, 931 Bensby, T., Feltzing, S., \& Oey, M. S. 2014, A\&A, 562, 71 Blanton, M. R., Bershady, M. A., Abolfathi, B., et al. 2017, AJ, 154, 28 Bovy, J., Rix, H.-W., Schlafly, E. F., et al. 2016, ApJ, 823, 30 Bullock, J. S., \& Johnston, K. V. 2005, ApJ, 635, 931

Carollo, D., Beers, T. C., Chiba, T. C., et al. 2010, ApJ, 712, 692 Carollo, D., Beers, T. C., Lee, Y. S., et al. 2007, Natur, 450, 1020 Carretta, E., Bragaglia, A., Gratton, R. G., et al. 2010, A\&A, 520, A95 Chiba, M., \& Beers, T. C. 2000, AJ, 119, 2843

De Silva, G. M., Freeman, K. C., Bland-Hawthorn, J., et al. 2015, MNRAS, 449, 2604

Eisenstein, D. J., Weinberg, D. H., Agol, E., et al. 2011, AJ, 142, 72

Ester, M., Kriegal, H. P., \& Sander, J. 1996, in Proc. 2nd Int. Conf. on Knowledge Discovery and Data Mining (KDD96) (Menlo Park, CA: AAAI Press), 226

Fernández-Alvar, E., Carigi, L., Allende Prieto, C., et al. 2017a, MNRAS, 465,1586

Fernández-Alvar, E., Carigi, L., Schuster, W. J., et al. 2017b, in press (arXiv:1711.06225)

Fishlock, C. K., Yong, D., Karakas, A. I., et al. 2017, MNRAS, 466, 4672

Font, A. S., Johnston, K. V., Bullock, J. S., \& Robertson, B. E. 2006, ApJ, 638, 585

Frebel, A., \& Norris, J. E. 2015, ARA\&A, 53, 631

García Pérez, A. E., Allende Prieto, C., Holtzman, J. A., et al. 2016, AJ, 151,144

Gilmore, G., Randich, S., Asplund, M., et al. 2012, Msngr, 147, 25

Gilmore, G., \& Reid, N. 1983, MNRAS, 202, 1025

Gratton, R., Sneden, C., \& Carretta, E. 2004, ARA\&A, 42, 385

Gratton, R. G., Carretta, E., Bragaglia, A., Lucatello, S., \& D’Orazi, V. 2010, A\&A, 517, A81

Gratton, R. G., Sneden, C., Carretta, E., \& Bragaglia, A. 2000, A\&A, 354, 169 Gunn, J. E., Siegmund, W. A., Mannery, E. J., et al. 2006, AJ, 131, 2332

Hartwick, F. D. A. 1987, in Proc. of the NATO Advanced Study Institute 207, The Galaxy (Dordrecht: Reidel), 281

Hasselquist, S., Shetrone, M., Smith, V., et al. 2017, ApJ, 845, 162

Hawkins, K., Jofré, P., Masseron, T., \& Gilmore, G. 2015, MNRAS, 453, 758 Hawkins, K., Masseron, T., Jofré, P., et al. 2016, A\&A, 594, A43

Holtzman, J. A., Shetrone, M., Johnson, J. A., et al. 2015, AJ, 150, 148

Ishigaki, M. N., Aoki, W., \& Chiba, M. 2013, ApJ, 771, 67

Ishigaki, M. N., Chiba, M., \& Aoki, W. 2012, ApJ, 753, 64

Jackson-Jones, R., Jofré, P., Hawkins, K., et al. 2014, A\&A, 571L, 5

Johnston, K. V. 2016, in IAU Symp. 317, The General Assembly of Galaxy Halos: Structure, Origin and Evolution (Cambridge: Cambridge Univ. Press), 1 
Karakas, A. I., \& Lattanzio, J. C. 2014, PASA, 31, e030

Kinman, T. D., Suntzeff, N. B., \& Kraft, R. P. 1994, AJ, 108, 1722

Law, D. R., \& Majewski, S. R. 2010, ApJ, 714, 229

Leaman, R., VandenBerg, D. A., \& Mendel, J. T. 2013, MNRAS, 436, 122

Lee, D. M., Johnston, K. V., Sen, B., \& Jessop, W. 2015, ApJ, 802, 48

Lee, Y. S., Beers, T. C., An, D., et al. 2011, ApJ, 738, 187

Lemasle, B., de Boer, T. J. L., Hill, V., et al. 2014, A\&A, 572, 82

Letarte, B., Hill, V., Tolstoy, E., et al. 2010, A\&A, 523, A17

MacQueen, J. 1967, in Proc. Fifth Berkeley Symp. on Math. Statist. and Prob 1, Statistics, ed. L. M. Le Cam \& J. Neyman (Berkeley, CA: Univ. of Calif. Press), 281

Majewski, S. R. 1993, ARA\&A, 31, 575

Majewski, S. R., Hasselquist, S., Łokas, E. L., et al. 2013, ApJL, 777, L13

Majewski, S. R., Nidever, D. L., Smith, V. V., et al. 2012, ApJL, 747, L37

Majewski, S. R., Schiavon, R. P., Frinchaboy, P. M., et al. 2017, AJ, 154, 94

Majewski, S. R., Skrutskie, M. F., Weinberg, M. D., \& Ostheimer, J. C. 2003, ApJ, 599, 1082

Martig, M., Fouesneau, M., Rix, H.-W., et al. 2016, MNRAS, 456, 3655

Minchev, I., Famaey, B., Quillen, A. C., et al. 2012, A\&A, 548, A127

Morrison, H. L. 1993, AJ, 105, 539

Mucciarelli, A., Bellazzini, M., Ibata, R., et al. 2017, A\&A, 605, A46

Navarro, J. F., Abadi, M. G., Venn, K. A., et al. 2011, MNRAS, 412, 1203

Nidever, D. L., Holtzman, J. A., Allende Prieto, C., et al. 2015, AJ, 150, 173

Nissen, P. E., \& Schuster, W. J. 1997, A\&A, 326, 751

Nissen, P. E., \& Schuster, W. J. 2010, A\&A, 511L, 10

Nissen, P. E., \& Schuster, W. J. 2011, A\&A, 530A, 15

Nomoto, K., Kobayashi, C., \& Tominaga, N. 2013, ARA\&A, 51, 457

Norris, J. E. 1994, ApJ, 431, 645

Quinn, P. J., Hernquist, L., \& Fullagar, D. P. 1993, ApJ, 403, 74

Ramírez, I., Meléndez, J., \& Chanamé, J. 2012, ApJ, 757, 164

Recio-Blanco, A., Rojas-Arriagada, A., de Laverny, P., et al. 2017, A\&A, 602, L14

Reddy, B. E., Lambert, D. L., \& Allende Prieto, C. 2006, MNRAS, 367, 1329
Rousseeuw, P. J. 1987, JCoAM, 20, 53

Ruchti, G. R., Fulbright, J. P., Wyse, R. F. G., et al. 2011, ApJ, 737, 9

Sbordone, L., Bonifacio, P., Buonanno, R., et al. 2007, A\&A, 465, 815

Schönrich, R. 2012, MNRAS, 427, 274

Schönrich, R., Binney, J., \& Dehnen, W. 2010, MNRAS, 403, 1829

Schuster, W. J., Moreno, E., Nissen, P. E., \& Pichardo, B. 2012, A\&A, 538, 21

Sellwood, J. A., \& Binney, J. J. 2002, MNRAS, 336, 785

Sheffield, A. A., Majewski, S. R., Johnston, K. V., et al. 2012, ApJ, 761, 161

Sommer-Larsen, J., \& Zhen, C. 1990, MNRAS, 242, 10

Souto, D., Cunha, K., Smith, V., et al. 2016, ApJ, 830, 35

Taylor, M. B. 2005, ASPC, 347, 29

Tissera, P. B., Scannapieco, C., Beers, T. C., \& Carollo, D. 2013, MNRAS, 432, 3391

Tissera, P. B., White, S. D. M., \& Scannapieco, C. 2012, MNRAS, 420, 255

Tolstoy, E., Hill, V., \& Tosi, M. 2009, ARA\&A, 47, 371

Tsujimoto, T., Nomoto, K., Yoshii, Y., et al. 1995, MNRAS, 277, 945

VandenBerg, D. A., Brogaard, K., Leaman, R., \& Casagrande, L. 2013, ApJ, 775,134

Van der Swaelmen, M., Hill, V., Primas, F., \& Cole, A. A. 2013, A\&A, 560,44

Venn, K. A., Irwin, M., Shetrone, M. D., et al. 2004, AJ, 128, 1177

Vera-Ciro, C., D’Onghia, E., Navarro, J., \& Abadi, M. 2014, ApJ, 794, 173

Wagner-Kaiser, R., Sarajedini, A., von Hippel, T., et al. 2017, MNRAS, 468, 1038

Walker, I. R., Mihos, J. C., \& Hernquist, L. 1996, ApJ, 460, 121

Yoshii, Y. 1982, PASJ, 34, 365

Zamora, O., García-Hernández, D. A., Allende Prieto, C., et al. 2015, AJ, 149,181

Zasowski, G., Johnson, J. A., Frinchaboy, P. M., et al. 2013, AJ, 146, 81

Zinn, R. 1993, in ASP Conf. Ser. 48, The Globular Cluster-Galaxy Connection, ed. G. H. Smith \& J. P. Brodie (San Francisco, CA: ASP), 38

Zinn, R. 1996, in ASP Conf. Ser. 92, Formation of the Galactic Halo...Inside and Out, ed. H. L. Morrison \& A. Sarajedini (San Francisco, CA: ASP), 211 